UCLA/93/TEP/49

hep-th/9312207

\title{
MANIFESTLY FINITE PERTURBATION THEORY FOR THE SHORT-DISTANCE EXPANSION OF CORRELATION FUNCTIONS IN THE TWO DIMENSIONAL ISING MODEL
}

\author{
Bakhtiar Mikhak and Amir M. Zarkesh \\ Physics Department \\ University of California, Los Angeles \\ Los Angeles, CA 90024, USA
}

\begin{abstract}
In the spirit of classic works of Wilson on the renormalization group and operator product expansion, a new framework for the study of the theory space of euclidean quantum field theories has been introduced. This formalism is particularly useful for elucidating the structure of the short-distance expansions of the $n$-point functions of a renormalizable quantum field theory near a non-trivial fixed point. We review and apply this formalism in the study of the scaling limit of the two dimensional massive Ising model. Renormalization group analysis and operator product expansions determine all the non-analytic mass dependence of the short-distance expansion of the correlation functions. An extension of the first order variational formula to higher orders provides a manifestly finite scheme for the perturbative calculation of the operator product coefficients to any order in parameters. A perturbative expansion of the correlation functions follows. We implement this scheme for a systematic study of correlation functions involving two spin operators. We show how the necessary non-trivial integrals can be calculated. As two concrete examples we explicitly calculate the short-distance expansion of the spin-spin correlation function to third order and the spin-spin-energy density correlation function to first order in the mass. We also discuss the applicability of our results to perturbations near other non-trivial fixed points corresponding to other unitary minimal models.
\end{abstract}

December 93

${ }^{*}$ Supported in part by D.O.E. contract DE-FG03-91ER 40662 Task C. 


\section{Introduction}

The equivalence of statistical mechanics models and quantum euclidean field theories with an ultraviolet cutoff has provided us with the exchange and deeper understanding of very fruitful notions, such as the renormalization group (RG) and spontaneous symmetry breaking, between the two disciplines. The modern renormalization group language offers a beautiful qualitative description of how the existence of second order (continuous) phase transitions in a statistical mechanical system is relevant in defining a local quantum field theory [- In particular the existence of a critical point and the number of parameters one needs to fine-tune in order to reach the critical point of a statistical model correspond to the existence of an ultraviolet fixed point and the number of relevant operators of the continuum field theory, respectively. In fact the exact solvability of statistical lattice models gives us detailed quantitative information on the existence and proper definition of the continuum limit of a quantum field theory and, in principle, allows for a non-perturbative evaluation of all the correlation functions in the theory円.

In many situations where the lattice model is not exactly solvable, the exact solvability of the fixed point theory is expected to allow for perturbative calculation of the correlation functions of the model near criticality. This is the situation ordinarily encountered in field theory calculations where the fixed point theory is trivial, i.e., a free field theory. It is reasonable to ask whether the same is true of non-trivial exactly solvable fixed point theories. Fixed point theories are the fixed points of the renormalization group action on the theory space of all quantum field theories. Due to the complexity of theory space with respect to the RG flows, information about theories not in the immediate neighborhood of a given ultraviolet fixed point is accessible only non-perturbatively. It is believed that, in many physically interesting cases, under the action of the renormalization group the theory flows from an ultraviolet (UV) fixed point to an infrared (IR) fixed point in the theory space.

It is very difficult to give a precise definition of the space of all quantum field theories. In fact this definition is dependent on the field-theoretic issues under consideration. For a better understanding of theory space it is clearly important to have a perturbative scheme for studying off-critical theories, in the vicinity of and away from their non-trivial fixed points, given the information encoded in the fixed point theories only. When off-critical theories are defined as perturbations of critical theories we should in general consider perturbations by relevant, marginal, and also irrelevant operators of the critical theory. Among these, the perturbations which are integrable are of special interest. Comparison of perturbative calculations in such models with exact results may shed light on how non-perturbative information can be extracted from such analysis.

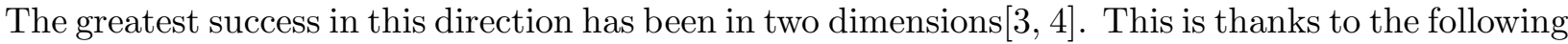
two facts. First, in two dimensions the scale invariance of a statistical system at its second order phase transition point, combined with rotational and translational invariance, implies full conformal invariance of the fixed point theory [5]. Second, there are many completely integrable field theories which can be regarded as perturbations of these critical models off criticality [4]. Therefore the space of two dimensional field theories, particularly conformal and integrable ones, provide the best theoretical playground for addressing many of the issues mentioned above.

Two dimensional conformal field theory, due to its central role in string theory [6] and possible classification of all two dimensional critical phenomena[7], has been studied extensively in the past

\footnotetext{
${ }^{1}$ For an example in two dimensions see[2].
} 
several yearst. Conformal field theories, besides describing critical statistical systems, give ultraviolet fixed points of the associated renormalizable quantum field theories. Though conformal invariance is enough to determine all correlation functions of the fixed point theories, these theories are by no means trivial. Unitary minimal models of BPZ[7], the simplest of which correspond to the critical and the tri-critical Ising models, are all examples of such theories. Even though these theories admit free field representations, they are still non-trivial, in light of the fact that conformal symmetries only admit coordinate space realizations 3 .

Certain perturbations of minimal models are known to be completely integrable. The simplest example of this is the thermal perturbation of the two dimensional Ising model. A much less trivial example is the RG flow between tri-critical and critical Ising models. It is believed that a suitable integrable perturbation of the tri-critical Ising model has the critical Ising model as its IR fixed point 3 , 9]. Such flows, aside from being physically interesting in their own right, provide useful toy models for higher dimensional low energy effective field theories of completely solvable high energy theories. These provide ample reason for a need for a calculational scheme which naturally takes advantage of the existence of non-trivial yet solvable fixed point theories. Even though there has been some success in doing perturbative calculations near non-trivial fixed points of specific models up to low orders, they have not lead to a systematic calculational scheme up to now.

Recently, expanding upon ideas originally put forth by Wilson [1, 10], and expanded upon by Wegner 11, 12], a new framework for the analysis of the theory space of all renormalizable $D$ dimensional euclidean quantum field theories has been introduced ([13]-[19]). Though this formalism is in principle non-perturbative, it provides a concrete program for perturbative analysis and calculation of the shortdistance expansion of the correlation functions of a renormalizable field theory around a non-trivial fixed point. The first step in this program is to make a particular choice of coordinates for the infinite dimensional theory space such that all but a finite number of coordinates vanish for renormalizable theories. With this choice we can analyze the structure of the renormalization group equations by counting the scale dimensions of the coordinates [13, 15].

The next step is to combine the renormalization group with the operator product expansion (OPE) and determine all non-analytic dependence on the renormalized parameters in the short-distance expansion of the $n$-point correlation functions [12]. At this point, all that remains for us to calculate are the RG invariant functions appearing in the solution of the RG equations 13. Their evaluation requires an explicit calculation based on a concrete regularization scheme, which was provided by the introduction of a variational formula for correlation functions in coordinate space[16]. In this formalism the operator product expansion is introduced and incorporated in the definition of the variational formula in order to regulate all the ultraviolet divergences. It is not necessary to establish the validity of the OPE by extensive calculations[10] as it is already built into the formalism (i.e., OPE is assumed).

The variational formula for the correlation functions realizes the derivative of correlation functions with respect to a parameter in terms of an insertion of a conjugate operator. The notion of conjugacy is the same as the one used in thermodynamics, in the sense that the expectation value of any such operator is defined to be the derivative of the free energy density of the system. The existence of such operators is an assumption. The variational formula is formulated in coordinate space, and involves divergent subtractions due to short-distance singularities of the renormalized theory. We also need to introduce finite counterterms to compensate for the arbitrariness of the subtraction scheme. The finite counterterms can be interpreted geometrically as connections in the theory space 16, 19.

\footnotetext{
${ }^{2}$ See articles in ref.[8] also.

${ }^{3}$ For these models the standard Feynman techniques are not applicable.
} 
The variational formula is very hard to derive from first principles and we can only demand its consistency with RG and the usual properties of derivatives. Requiring consistency with the RG equations leads to a relationship between the anomalous dimensions of the composite operators and the unintegrable part of the operator product expansion [16]. The curvature of the above connection appears in this relationship naturally. The commutativity of mixed higher order derivatives (Maxwell relations) leads to an expression for the curvature of the connection in terms of a double integral over a finite domain. This provides us with a very natural setting for studying issues relating to general covariance and geometry in theory space [18, 19. Upon applying the variational formula for correlation functions to the OPE we arrive at a variational formula for the operator product coefficients[17]. Repeated application of these variational formulas allows for a perturbative calculation of the correlation functions.

The large number of exact results in two dimensional conformal and integrable field theories motivates the application of the above formalism to the study of the space of two dimensional quantum field theories. Since the above framework and its full conceptual and calculational power is not yet popular we decided to present a comprehensive discussion of the systematic calculational scheme which is contained in this formalism. In particular we illustrate the usefulness of this scheme in analyzing a historically significant model, the two dimensional Ising model. This model corresponds to the best understood non-trivial fixed point in the space of two dimensional field theories. It was solved exactly nearly half a century agot, and has served as an important toy model for the study of existence and definition of continuum field theories starting from lattice models [2]. The correlation functions of the model are known exactly, thanks to its complete integrability at and away from the fixed point [2, 7, 25]. The short- and long-distance expansions of the correlation functions encode the operator content of the UV and IR fixed point theories. It is desirable to understand the structure of these expansions field-theoretically. Therefore, our interest in this model is twofold. First, since the presence of the spin field in the Ising model does not allow us to apply the traditional Feynman approach to perturbative calculations in field theory, the use of the new formalism is essential. Second, we can check the results of our calculations againt the available exact results. The crucial point is that this method is in principle equally powerful for many other interesting models for which exact solutions are not known.

The purpose of the present paper is to discuss the formalism sketched above in a general renormalizable field theory with a UV fixed point, and to extend the proof of infrared finiteness of the variational formula for the operator product coefficients to any order. This essentially amounts to the consistency of the variational formula with the analyticity of the OPE coefficients and leads to a manifestly finite variational formula for general operator product coefficients ? We therefore arrive at a systematic method for the perturbative calculation of OPE coefficients, and consequently correlation functions, in the vicinity of any non-trivial ultraviolet fixed point. As an application of our results we calculate the spin-spin and the spin-spin-energy density correlation functions in the massive Ising model perturbatively.

This paper not only provides the conceptual and calculational details of our previous work [26], but also extends the calculational framework of ref. [16, 17] to higher orders. Our manifestly finite calculational scheme is a product of a complete examination of the infrared structure of the higher order variational formulas, and demanding consistency with locality principles. Calculation of non-trivial twoand three-point functions in the two dimensional Ising model within this scheme are explicit examples of the practicality of this formalism in addition to its conceptual clarity. These are the first field-theoretic

\footnotetext{
${ }^{4}$ For the Onsager solution of the Ising model, see ref. 20] and references therein.

${ }^{5}$ These are the coefficients appearing in the expansion of correlation functions in terms of expectation value of single operators.
} 
calculations near a non-trivial fixed point 9 and should, in principle, be generalizable to many other two dimensional models. The main obstacle for carrying out the same analysis for other models is finding a systematic way of calculating the necessary finite integrals, which are quite complicated in general. However, our success in finding a systematic technique for calculating the integrals for the Ising model correlation functions gives us hope that the same should be possible at least for other unitary minimal models.

The organization of the paper is as follows. In section 2 we review the RG analysis of refs. 13 - 15 in general and in the two dimensional Ising model. In section 3 we review the variational formulas [16, 17] and discuss their ultraviolet and infrared divergence structures in detail. We also introduce a variational formula for general operator product expansion coefficients in this section. The consistency of the variational formula with the differentiability of the OPE coefficients to a given order is shown to be equivalent to the IR finiteness of the variational formula to that order. Hence, the analyticity of the OPE coefficients implies that the variational formula is manifestly free of divergences to any order. In section 4 we analyze the UV structure of the variational formulas for the Ising model. Here we fix our choice of the parameters used in the remainder of the paper. In section 5 we apply the results of section 3 to the Ising model. We describe the structure of the $n \underline{\text { th }}$ order correction to the scaling behavior of the short-distance expansion of the spin-spin correlation function, and explain how the required integrals can be performed. For completeness, the details of the explicit calculation to third order 26] are presented in appendix B. In section 6, as another non-trivial application of our results, we discuss the calculation of the spin-spin-energy density correlation function to first order. In section 7 we present our conclusions. In appendix A some relevant facts about the operator content and the correlation functions of the critical Ising model are presented.

\section{Renormalization Group Analysis}

\subsection{General Theory}

The Action of the Renormalization Group on the Theory Space

In the Wilsonian approach to renormalization we consider the theory space $(T S)$ of all regularized quantum field theories in $D$ dimensional euclidean space 1]. The theories in $T S$ are regularized with an ultraviolet cutoff $a$ which is assumed to be invariant under both rotations and translations. The cutoff may be taken to be a lattice unit as long as the continuum theory obtained in the limit of vanishing lattice spacing is rotationally and translationally invariant. Let $\mathcal{H}$ denote a point in the theory space representing the action of a $D$ dimensional euclidean quantum field theory 7 .

The action of the renormalization group $(\mathrm{RG})$, denoted by $\mathcal{R}(\ell)$, is parametrized by a real variable $\ell$ and is defined as an integration over fluctuations between $a$ and $a e^{-\ell}$ followed by a dilation by a factor $e^{-\ell}$. Hence, the distance $r$, measured in units of the cutoff, is transformed to $r e^{-\ell}$, while the renormalization point $r=1$ is fixed. Note that RG acts toward the infrared and differs from the standard definition by rescaling. We generally assume the existence of fixed points in $T S$ with respect to the RG transformations. Let $\mathcal{H}^{*}$ denote one such point defined with the property

\footnotetext{
${ }^{6}$ Dotsenko 27] has calculated the second order correction to the spin-spin correlation function, but, as he points out, the generalization of his analytical continuation method to other models seems formidable.

${ }^{7}$ Equivalently $\mathcal{H}$ can be regarded as the hamiltonian of a $D$ dimensional statistical system.
} 


$$
\mathcal{R}(\ell) \mathcal{H}^{*}=\mathcal{H}^{*} \quad(\forall \ell \in \mathrm{R})
$$

At the fixed point scale invariance implies

$$
\left\langle\bar{\phi}_{i_{1}}\left(\lambda r_{1}\right) \cdots \bar{\phi}_{i_{n}}\left(\lambda r_{n}\right)\right\rangle_{\mathcal{H}^{*}}=\frac{1}{\lambda^{\left(x_{i_{1}}+\cdots+x_{i_{n}}\right)}}\left\langle\bar{\phi}_{i_{1}}\left(r_{1}\right) \cdots \bar{\phi}_{i_{n}}\left(r_{n}\right)\right\rangle_{\mathcal{H}^{*}}
$$

and we can assume that the operators $\bar{\phi}_{i}$ have non-negative scale dimensions $x_{i}$, and are called relevant, marginal, or irrelevant if $x_{i}$ are smaller than, equal to, or greater than $D$, respectively.

The hamiltonian of a theory in the neighborhood of $\mathcal{H}^{*}$ is obtained by adding a linear combination of local scalar ${ }^{\beta}$ operators to the fixed point hamiltonian. Let $\left\{\bar{\phi}_{i}\right\}$ denote a complete basis of local scalar operators which are infinite in number. The generalized mass parameters $\left\{\mu^{i}\right\}$, introduced by Wilson, are the coefficients of these local scalar operators and provide local coordinates for the TS. Hence, $T S$ is infinite dimensional.

Generally, starting from any hamiltonian $\mathcal{H}$ in the neighborhood of $\mathcal{H}^{*}$, RG transformations $\mathcal{R}(\ell)$ generate a trajectory of hamiltonians $\mathcal{R}(\ell) \mathcal{H}$ parametrized by $\ell$. The range of $\ell$ can be extended in both directions as long as the trajectory stays within the theory space. Not all trajectories can be extended without limit. A physically interesting subspace of $T S$ is the space of renormalized trajectories, denoted by $S(\infty)$, consisting of all trajectories which can be traced back to $\ell=-\infty$ without going out of the theory space. I] Renormalizable theories are typically characterized by a finite number of renormalized parameters and we should therefore be able to parametrize $S(\infty)$ by a finite number of parameters forming a finite dimensional subspace of the theory space, accessible asymptotically in the $\ell \rightarrow \infty$ limit of the RG flows. Let us explain these points more carefully[1, 21].

Consider the lattice models parametrized by couplings $\kappa^{i} \mathbb{W}$ where $i \in \mathcal{I}$ with $\mathcal{I}$ an (in general infinite) index set. Assume that the model undergoes a second order phase transition at $\kappa^{(c) i}$ parametrizing the critical hamiltonian $\mathcal{H}_{\text {lattice }}^{(c)}$. At the critical point all parameters have well-defined scale dimensions, $y_{i}=D-x_{i}$, and we assume that only a finite number of them have positive scale dimensions. Starting from an almost critical theory, under RG transformations the effect of the couplings with negative scale dimensions gets washed out. Therefore in the infrared limit the action of the renormalization group singles out a finite dimensional subspace of the theory space corresponding to a renormalized trajectory, i.e., universality. The dimension of this subspace is equal to the number of relevant operators conjugate to couplings with positive scale dimensions.

When the continuum theory exists, with the proper choice of coordinates (which will be described shortly) $\mathcal{H}_{\text {lattice }}^{(c)}$ flows to the origin of this subspace corresponding to $\mathcal{H}^{*}$ which is the UV fixed point of a renormalized field theory originating from it. In practice after reaching the renormalized trajectory in the infrared limit we can trace back to $\mathcal{H}^{*}$ along the renormalized trajectory $\square$. Therefore these theories are defined up to infinitesimal distances and on the trajectory the parameter $a$ plays the role of a renormalization point. In the process of defining the continuum limit in this way we are led to considering a finite number of renormalized parameters, $g^{i}$, characterizing this trajectory, which are analytic functions of the couplings $\kappa^{i}$. Therefore the goal would be to find a particular choice of

\footnotetext{
${ }^{8}$ Thanks to the assumption of rotational invariance we need to only consider scalar operators.

${ }^{9}$ This is equivalent to the assumption of the existence of a UV fixed point at the origin of renormalized trajectories.

${ }^{10}$ This is one particular choice for Wilson's generalized mass parameters.

${ }^{11}$ Since proving the existence of UV fixed points is in general very difficult, we assume it. In principle these fixed point theories are fully interactive.
} 
renormalized parameters, which allows for the study of the non-analytic dependence on the generalized mass parameters in the short-distance expansion of the correlation functions. In the next subsection we will see how this happens for the Ising model.

While Wilson's generalized mass parameters provide local coordinates for the infinite dimensional theory space, they are not necessarily the most suitable choice for a smooth parametrization of the renormalized trajectories. First, since we only need a finite number of renormalized parameters to parameterize the renormalized trajectories, we would expect that there must exist a new set of coordinates for $T S$ such that all but a finite number of them vanish on $S(\infty)$. Second, it would be desirable to be able to analyze the structure of the RG equations for these new coordinates by counting scale dimensions only. The advantage of these new coordinates would be that their scale dimensions are additively conserved under RG flows. This, in addition to the analyticity requirement of the RG equations implied by the locality of the theory, restricts the form of the RG equations to contain mixing with parameters of lower or equal scale dimensions only.

Based on Wegner's scaling fields [11], it has been shown that the analytic transformation of interest to the new coordinates $g^{i}(i=1 \cdots N)$ exists [13]. Under this change of coordinates, which is assumed to be analytic, our basis of local operators transforms like a vector. Denote the new basis of composite operators by $\left\{\phi_{i}\right\}_{g}$. In terms of these new coordinates, the operators $\phi_{i}$ have well-defined short-distance singularities only for the theories on $S(\infty)$. This allows for a systematic determination of the corrections to the scaling behavior of the short-distance expansion of the correlation functions. Note that if we consider general theories not restricted to $S(\infty)$, the correlation functions contain arbitrarily high singularities, even in terms of the new coordinates.

The coordinates of the theory space can be chosen such that the action of the renormalization group on the renormalized trajectories is

$$
\frac{d}{d \ell} g^{i}=\beta^{i}(g) \equiv y_{i} g^{i}+\sum_{\substack{I ; y_{i_{1}}, \cdots, y_{i_{n}} \geq 0 \\ y_{I}=y_{i}}} \frac{1}{n !} \beta_{i, I} g^{I}
$$

where $I$ stands for $i_{1} \cdots i_{n}, y_{I}=y_{i_{1}}+\cdots+y_{i_{n}}$ and $g^{I} \equiv g^{i_{1}} \cdots g^{i_{n}}$. The coefficients $\beta_{i, I}$ are independent of $g$. Note that $g=0$ is kept invariant under (2.3). In ref. 13. it was shown that with this choice of coordinates the short distance limit reduces to the limit of $g^{i} \rightarrow 0$.

Vector Bundle of Composite Operators

Each point $g$ in the space of renormalizable theories is a renormalized field theory which has an infinite number of linearly independent composite fields. $\left\{\phi_{a}\right\}_{g}$ denotes a complete basis of composite fields at $g$. The space of composite fields is a linear space, and therefore forms an infinite dimensional vector bundle over $S(\infty)$ 19. The choice of the local basis is by no means unique, and two bases $\left\{\phi_{a}\right\}_{g}$ and $\left\{\phi_{a}^{\prime}\right\}_{g}$ are related by an invertible infinite dimensional matrix $N(g)$ via

$$
\phi_{a}^{\prime}=(N(g))_{a}^{b} \phi_{b}
$$

The physical quantities, the correlation functions of $n$ composite fields $\left\langle\phi_{a_{1}}\left(r_{1}\right) \cdots \phi_{a_{n}}\left(r_{n}\right)\right\rangle_{g}$, form rank-n tensors on the theory space.

Among the infinite number of composite fields, $N$ composite scalar fields stand out; they are the fields $\mathcal{O}_{i}$ conjugate to the parameters $g^{i}$. The notion of conjugacy should be familiar from thermody- 
namics. The derivative of the free energy density $F(g)$ with respect to $g^{i}$ is given by the expectation value of $\mathcal{O}_{i}$ [2]:

$$
\left\langle\mathcal{O}_{i}\right\rangle_{g}=\frac{\partial}{\partial g^{i}} F(g)
$$

The RG action (2.3) combined with canonical scaling of the free energy density

$$
\frac{d}{d \ell} F(g)=D F(g)
$$

and (2.5) induces an RG equation for composite operators $\mathcal{O}_{i}$ :

$$
\frac{d}{d \ell} \mathcal{O}_{i}=D \mathcal{O}_{i}-\frac{\partial \beta^{j}}{\partial g^{i}} \mathcal{O}_{j}
$$

The particular choice of parameters $\left\{g^{i}\right\}$ restricts the form of RG equations for the general composite fields; scale dimensions are additively conserved, and under RG any field can only mix with operators of equal or smaller scale dimensions, with mixing coefficients analytic in the parameters. Therefore, introducing an infinite column vector $\Phi$ of composite operators, we can write

$$
\frac{d}{d \ell} \Phi=\Gamma(g) \Phi
$$

where the matrix

$$
\Gamma_{a}^{b}(g) \equiv x_{a} \delta_{a}^{b}+O(g)
$$

gives the scale dimensions $x_{a}$ and the anomalous dimensions of renormalized fields. The precise meaning of the above RG equations is given by the following RG equations for the correlation functions:

$$
\begin{aligned}
\frac{d}{d \ell}\left\langle\phi_{a_{1}}\left(r_{1}\right) \cdots \phi_{a_{n}}\left(r_{n}\right)\right\rangle_{g} & \equiv\left(-\sum_{k=1}^{n} r_{k, \mu} \frac{\partial}{\partial r_{k, \mu}}+\beta^{i} \frac{\partial}{\partial g^{i}}\right)\left\langle\phi_{a_{1}}\left(r_{1}\right) \cdots \phi_{a_{n}}\left(r_{n}\right)\right\rangle_{g} \\
& =\sum_{k=1}^{n} \Gamma_{a_{k}}^{b}(g)\left\langle\phi_{a_{1}}\left(r_{1}\right) \cdots \phi_{b}\left(r_{k}\right) \cdots \phi_{a_{n}}\left(r_{n}\right)\right\rangle_{g} .
\end{aligned}
$$

\section{Operator Product Expansion}

The operator product expansion (OPE) plays a central role in the analysis of the short-distance expansion of correlation functions 10. Wegner combined OPE with the RG equations to ascribe all non-analytic dependence on the scaling fields to the vacuum expectation values of single composite operators 12]. When $r$ defined by $r^{2} \equiv \sum_{i<j}\left(r_{i}-r_{j}\right)^{2}$, is small compared with the correlation length, we can write

\footnotetext{
${ }^{12}$ In order to make the notion of conjugacy in field theories precise, we also need to specify how the derivative of a correlation function is realized. The variational formula (VF) in section 3 is one such realization.
} 


$$
\phi_{a_{1}}\left(r_{1}\right) \cdots \phi_{a_{n}}\left(r_{n}\right)=C_{a_{1} \cdots a_{n}}{ }^{b}\left(r_{1}, \cdots, r_{n} ; g\right) \phi_{b}(0)
$$

where $C_{a_{1} \cdots a_{n}}{ }^{b}$ is invariant under translation. The validity of this equation as an operator equation requires the sum over $b$ to run over all local operators with or without vanishing spins. This is understood as an asymptotic expansion of the correlation functions

$$
\left\langle\phi_{a_{1}}\left(r_{1}\right) \cdots \phi_{a_{n}}\left(r_{n}\right) \phi_{d_{1}}\left(\xi_{1}\right) \cdots \phi_{d_{k}}\left(\xi_{k}\right)\right\rangle_{g}=C_{a_{1} \cdots a_{n}}{ }^{b}\left(r_{1} \cdots r_{n} ; g\right)\left\langle\phi_{b}(0) \phi_{d_{1}}\left(\xi_{1}\right) \cdots \phi_{d_{k}}\left(\xi_{k}\right)\right\rangle_{g}
$$

The fundamental assumption for the operator product expansion is that the coefficients $C_{a_{1} \cdots a_{n}}{ }^{b}(g)$ depend on the parameters only analytically. This is equivalent to the local nature of the operator product expansion: short-distance physics alone guarantees (2.12) to be valid.

Using the RG equation (2.8) we can derive the RG equation for the coefficient functions

$$
\frac{d}{d \ell} C_{a_{1} \cdots a_{n}}{ }^{b}\left(r_{1}, \cdots, r_{n} ; g\right)=-C_{a_{1} \cdots a_{n}}{ }^{d} \Gamma_{d}^{b}(g)+\sum_{i=1}^{n} \Gamma_{a_{i}}{ }^{d}(g) C_{a_{1} \cdots a_{i-1} d a_{i+1} \cdots a_{n}}{ }^{b}
$$

where the matrix $\Gamma(g)$ is given by (2.9).

$\underline{\text { Solution of RG Equations }}$

In order to solve RG equations (2.10) and (2.13) we define the running parameters $\bar{g}(\ell ; g)$ as the solutions of RG equations

$$
\frac{\partial}{\partial \ell} \bar{g}^{i}(\ell ; g)=\beta^{i}(\bar{g}(\ell ; g))
$$

with the initial conditions $\bar{g}^{i}(0 ; g)=g^{i}$. We will suppress these initial conditions from now on, and we will denote $\bar{g}^{i}(\ell ; g)$ simply by $\bar{g}^{i}(\ell)$. We can then construct $N$ RG invariant quantities, $\bar{g}^{i}(\ln r)$. The $\mathrm{RG}$ invariance follows from the fact that the dependence on the change of the coordinate $r$ under the $\mathrm{RG}$ is cancelled by the dependence on the change of initial parameters $g$ under the RG.

We also need to introduce the matrix $G(r ; g)$ that satisfies

$$
\frac{d}{d \ell} G(r ; g)=\Gamma(g) G(r ; g)
$$

with the initial condition $G(1 ; g)=\mathbf{1}$. The matrix $G(r ; g)$ is easily calculable for any field theory with at most one marginal parameter. Using this matrix the solution to equation 2.13) is

$$
C_{a_{1} \cdots a_{n}}{ }^{d}\left(r_{1}, \cdots, r_{n} ; g\right)=G_{a_{1}}^{b_{1}}(r ; g) \cdots G_{a_{n}}^{b_{n}}(r ; g) H_{b_{1} \cdots b_{n}}{ }^{e}\left(\frac{r_{i}-r_{j}}{r} ; \bar{g}(\ln r)\right)\left(G^{-1}\right)_{e}^{d}(r ; g),
$$

where 


$$
H_{a_{1} \cdots a_{n}}{ }^{d}\left(\frac{r_{i}-r_{j}}{r} ; g\right)=C_{a_{1} \cdots a_{n}}{ }^{d}\left(\frac{r_{1}}{r}, \cdots, \frac{r_{n}}{r} ; g\right)
$$

is an analytic function of $g$, due to the analyticity assumptions for $C_{a_{1} \cdots a_{n}}{ }^{d}(g)$. Therefore the solution of (2.10) is

$$
\left\langle\phi_{a_{1}}\left(r_{1}\right) \cdots \phi_{a_{n}}\left(r_{n}\right)\right\rangle_{g}=G_{a_{1}}^{b_{1}}(r ; g) \cdots G_{a_{n}}^{b_{n}}(r ; g) H_{b_{1} \cdots b_{n}}{ }^{d}\left(\frac{r_{i}-r_{j}}{r} ; \bar{g}(\ln r)\right)\left\langle\phi_{d}\right\rangle_{\bar{g}(\ln r)},
$$

and all the non-analytic dependence on $g$ 's in the correlation functions comes from the expectation values of single operators (2.5) through the non-analyticity of the free energy density.

Since the short-distance limit on renormalized trajectories is equivalent to the limit $g \rightarrow 0$, we obtain the short distance behavior [13]

$$
\left\langle\phi_{a_{1}}\left(r_{1}\right) \cdots \phi_{a_{n}}\left(r_{n}\right)\right\rangle_{g} \rightarrow\left\langle\phi_{a_{1}}\left(r_{1}\right) \cdots \phi_{a_{n}}\left(r_{n}\right)\right\rangle_{\mathcal{H}^{*}}
$$

which is consistent with our previous comment that the operators $\phi_{i}$ have well-defined short distance singularities only for the theories on $S(\infty)$.

\subsection{The Two Dimensional Ising Model}

The Ising model is defined on a periodic lattice by the hamiltonian

$$
\mathcal{H}_{\text {lattice }} \equiv-K \sum_{m, n=0}^{N}\left\{\sigma_{m, n} \sigma_{m+1, n}+\sigma_{m, n} \sigma_{m, n+1}\right\} \text {, }
$$

where $\sigma_{m, n}= \pm 1$. This theory undergoes a second order phase transition at $K=K_{c}$ given by

$$
\sinh \left(2 K_{c}\right)=1
$$

The modern renormalization group language offers a qualitative description of continuous phase transitions. Let us describe the phase transition in the Ising model in this language. $\mathcal{H}_{\text {lattice }}$ defines a one parameter family of theories in the theory space. The action of the renormalization group, in the infrared limit, singles out a renormalized trajectory in the theory space such that theories with

$K<K_{c}$ and $K>K_{c}$ flow to two different tails of this line. The critical theory $\mathcal{H}_{\text {lattice }}^{(c)}$, for $K=K_{c}$, flows to $\mathcal{H}^{*}$ (the origin of the coordinates on the renormalized trajectories) along a critical line. On the renormalized trajectory, the vicinity of the origin characterizes the short-distance behavior of the scaling limit of the lattice theory. This limit is defined by letting $\epsilon \equiv 4\left(K_{c}-K\right) \rightarrow 0$, but keeping the product $t=\epsilon R$ ( $R$ is the distance in lattice units) finite. The renormalized mass $m$ should be proportional to $\epsilon$ up to first order. The complete dependence of $m$ on $\epsilon$ is fixed by demanding a simple RG equation for $m$. 
In a statistical mechanical system near its second order phase transition point, where the correlation length diverges, information about the details of the lattice is washed out. If the above scaling limit exists we have a well-defined quantum field theory all the way up to zero distance. This fact is usually stated as the existence of an unstable UV fixed point which can be reached backward along the renormalized trajectory, using the RG equations for the renormalized parameters. We recover rotational invariance in the continuum limit, and the theory is equivalent to a theory of a free massive Majorana fermion. The theory is parametrized by the cosmological constant $g_{1}$ and the mass $m$, which are conjugate to the operators $\mathbf{1}$ and $\mathcal{O}_{m}$ (corresponding to the energy density operator $i \bar{\psi} \psi$ ), respectively. These parameters satisfy the following $\mathrm{RG}$ equations

$$
\begin{gathered}
\frac{d}{d \ell} g_{\mathbf{1}}=2 g_{\mathbf{1}}+\frac{1}{2} \beta_{\mathbf{1}} m^{2} \\
\frac{d}{d \ell} m=m .
\end{gathered}
$$

There are two RG invariant quantities

$$
\begin{gathered}
\bar{g}_{\mathbf{1}}(\ln r)=r^{2} g_{\mathbf{1}}+\frac{1}{2} \beta_{\mathbf{1}} m^{2} r^{2} \ln r \\
\bar{m}(\ln r)=m r .
\end{gathered}
$$

In this case (2.7) reduces to

$$
\frac{d}{d \ell} \mathcal{O}_{m}=\mathcal{O}_{m}-\beta_{\mathbf{1}} m \mathbf{1}
$$

Taking the expectation value of both sides and using $(2.23)$ we have

$$
\left\langle\mathcal{O}_{m}\right\rangle_{m}=-\beta_{\mathbf{1}} m \ln (m c)
$$

where $\mathrm{c}$ is a constant of integration. Equations (2.27) and (2.5) give

$$
F\left(g_{\mathbf{1}}, m\right)=g_{\mathbf{1}}-\frac{1}{2} \beta_{\mathbf{1}} m^{2} \ln (m c)+\frac{1}{4} \beta_{\mathbf{1}} m^{2}
$$

This was one of the main results of ref. [14].

Since $g_{1}$ only contributes an additive constant to the free energy density, none of the correlation functions of local operators depend on it. In this case, we can effectively regard $S(\infty)$ as a line parametrized by a real parameter $m$. This is the line corresponding to the theory of a free massive Majorana fermion mentioned above. Therefore from now on all of our physical quantities will depend on $m$ only.

The RG analysis of one point functions of more general composite operators in the Ising model is quite straightforward. The first reason for this, as shown in appendix A, is that the couplings to all the members of class $\sigma$ can be turned off, due to the locality of the RG transformations and the special OPE structure of the operators of the critical Ising model (see A.7). The second reason is that, due 
to the additive conservation of the scale dimension of operators under RG transformations, the matrix $\Gamma(m)$ has the following structure

$$
\begin{aligned}
\Gamma_{a}{ }^{b}(m) & =x_{a} \delta_{a}{ }^{b}+\gamma_{a}{ }^{b} m^{x_{a}-x_{b}} & & \text { iff } x_{a}-x_{b} \in \mathbb{Z}^{+} \\
& =x_{a} \delta_{a}{ }^{b} & & \text { otherwise. }
\end{aligned}
$$

Note that (2.29a) is particularly simple since there is no marginal (i.e., dimension 2) scalar operator in this theory. Using $(2.23)$ and (2.29a) we can write $(2.8)$ as

$$
\frac{d}{d \ell}\left[m^{-X}\langle\Phi\rangle_{m}\right]=\gamma\left[m^{-X}\langle\Phi\rangle_{m}\right]
$$

where we have introduced the infinite dimensional diagonal matrix $X \equiv \operatorname{diag}\left[x_{i}\right]$.

Now since we are considering a unitary model, the lowest dimension operator is the identity operator with scale dimension zero. Arrange the column vector $\Phi$ such that operators with higher scale dimensions are lower in the column. With this choice the matrix $\gamma$ is lower triangular. Therefore upon repeated differentiation of the above we get a non-zero result

$$
\frac{d^{n}}{d \ell^{n}}\left[m^{-x_{a}}\left\langle\phi_{a}\right\rangle_{m}\right]=\gamma_{a}^{b_{1}} \gamma_{b_{1}}^{b_{2}} \cdots \gamma_{b_{n-1}}^{b_{n}}\left[m^{-x_{b_{n}}}\left\langle\phi_{b_{n}}\right\rangle_{m}\right]
$$

if and only if $a>b_{1}>\cdots>b_{n}$. We therefore see that the case $\phi_{b_{n}}=\mathbf{1}$ corresponds to the largest value $n$ can have. Using equation (2.23) we see that $n$ is the highest power of $\ln (m c)$ and is equal to the number of scale dimensions smaller than $x_{a}$, where at least one non-derivative scalar composite operator exists. We therefore have

$$
\left\langle\phi_{a}\right\rangle_{m}=m^{x_{a}} \frac{(-1)^{n}}{n !} \gamma_{a}^{b_{1}} \gamma_{b_{1}}{ }^{b_{2}} \cdots \gamma_{b_{n-1}} b_{n}[\ln (m c)]^{n}+\cdots
$$

Recall that we can change our basis of local operators, which is equivalent to an analytic change of coordinates in the neighborhood of renormalized trajectories. In other words, this transformation should not spoil the mass independence of the RG equations

$$
\tilde{\phi}_{a}=\phi_{a}+\sum_{b: x_{a}>x_{b}} n_{a}^{b} m^{x_{a}-x_{b}} \phi_{b} \equiv N_{a}^{b}(m) \phi_{b}
$$

such that with $\tilde{\gamma}=N(m) \gamma N^{-1}(m)$ we can write:

$$
\left\langle\tilde{\phi}_{a}\right\rangle_{m}=m^{x_{a}} \frac{(-1)^{n}}{n !} \tilde{\gamma}_{a}{ }^{b_{1}} \tilde{\gamma}_{b_{1}}^{b_{2}} \ldots \tilde{\gamma}_{b_{n-1}}^{b_{n}}[\ln (m c)]^{n} .
$$

This transformation exists if a reduced matrix, obtained from $\gamma$ upon eliminating the entries due to $\mathbf{1}$, is diagonalizable.

For the RG analysis of more complicated correlation functions we need to know the matrix $G(r ; m)$. In fact we can solve (2.15) very easily to get 


$$
G(r ; m)=m^{X} r^{-\gamma}(m r)^{-X}
$$

where $\gamma_{a}^{b}$ are non-vanishing only if $x_{a}-x_{b}$ is a positive integer. With the explicit form of $G(r ; m)$ at hand, the correlation functions (2.18) are determined up to RG invariant functions. The remainder of this paper is concerned with perturbative calculation of these RG invariant functions.

\section{Perturbation Theory for OPE Coefficients}

As we have seen in equation (2.16), after separating out the non-analyticities of correlation functions encoded in expectation values of single operators, we remain with the OPE coefficients which are analytic functions of parameters. Renormalization group analysis fixes these analytic functions up to RG invariant combinations 2.17). Determination of these functions requires explicit perturbative calculations, based on a concrete regularization scheme. Note that, since the OPE coefficients are universal, they do not depend on how we calculate them.

On the other hand, conjugacy in thermodynamics and statistical mechanics is an exact notion, irrespective of any approximation technique used in practical calculations. The thermodynamical definition of conjugacy is that the derivative of a correlation function with respect to a parameter is given by an insertion of the volume integral of the corresponding conjugate operator. As we already discussed, well-defined renormalizable field theories are defined as the scaling limit of statistical systems. In taking this limit, physical short-distance singularities appear, which prevent us from naively borrowing the definition of conjugacy as given in thermodynamics. In order to extend this definition to field theories, the notion of singular operator product expansion is introduced to cure the physical ultraviolet singularities mentioned above. Moreover, the full operator product expansion (both singular and integrable) need to be introduced to incorporate locality into this formalism.

The variational formula for correlation functions gives a realization of the derivative with respect to a renormalized parameter which satisfies the requirements imposed by the field-theoretical conjugacy relation [16]. Consistency of this variational formula with the locality of the operator product expansion amounts to the infrared finiteness of the variational formula for the operator coefficients. The latter variational formula has been introduced and proven to be infrared finite in ref.[17]. We provide the details of the proof of the infrared finiteness to all orders. As a by-product we arrive at a manifestly finite calculational scheme for the perturbative calculation of the OPE coefficients. In the following sections we discuss these issues in detail.

\subsection{Variational Formula for Correlation Functions}

In order to characterize the short-distance singularities of the renormalized correlation functions, whose volume integral will be needed shortly, and to choose the suitable subtractions to make these integrals UV finite, consider the following singular OPE coefficients 


$$
\mathcal{O}_{i}(r) \phi_{a}(0)=\bar{C}_{i a}^{(s) b}(r ; g) \phi_{b}(0)+O\left(\frac{1}{r^{D}}\right)
$$

Here, we assume the average over the orientation of the coordinate vector $r_{\mu}$ has been taken, such that the right hand side includes only the scalar composite operators. Also note that only composite fields $\phi_{b}$ whose scale dimension are at most $x_{i}+x_{a}-D$ should be kept; we only need to consider the unintegrable part of the OPE coefficients for calculating the subtractions.

The divergences arising from the singular OPE coefficients are physical short-distance singularities and they exist even after renormalization. By using these singular OPE coefficients, the following variational formula has been introduced [16]

$$
\begin{aligned}
-\partial_{i}\left\langle\phi_{a_{1}}\left(r_{1}\right) \cdots \phi_{a_{n}}\left(r_{n}\right)\right\rangle_{g}=\lim _{\epsilon \rightarrow 0}\left[\int_{\left|r^{\prime}-r_{k}\right| \geq \epsilon} d^{D} r^{\prime}\left\langle\mathcal{O}_{i}^{c}\left(r^{\prime}\right) \phi_{a_{1}}\left(r_{1}\right) \cdots \phi_{a_{n}}\left(r_{n}\right)\right\rangle_{g}\right. \\
\left.\quad+\sum_{k=1}^{n} A_{i a_{k}}{ }^{b}(g ; \epsilon)\left\langle\phi_{a_{1}}\left(r_{1}\right) \cdots \phi_{b}\left(r_{k}\right) \cdots \phi_{a_{n}}\left(r_{n}\right)\right\rangle_{g}\right]
\end{aligned}
$$

where $\partial_{i} \equiv \frac{\partial}{\partial g_{i}}$, the definition of connected operators is

$$
\mathcal{O}_{i}^{c}(r) \equiv \mathcal{O}_{i}(r)-\left\langle\mathcal{O}_{i}\right\rangle_{g}
$$

and the definition of the subtractions, in the matrix notation, is

$$
A_{i}(\epsilon ; g) \equiv-\int_{1 \geq r \geq \epsilon} d^{D} r \bar{C}_{i}^{(s)}(r ; g)+c_{i}(g)
$$

Here 1 is the renormalization point, fixed under the renormalization group.

The arbitrariness involved in the subtraction of the divergent counterterms is compensated for by the introduction of finite counterterms $c_{i}(g)$. These finite counterterms, in addition to the above mentioned physically important role, have a natural geometrical meaning in the theory space. The correlation functions are tensor fields on the space of renormalized trajectories, and therefore their partial derivatives are not covariant quantities. To be able to define a covariant derivative we need to have a connection on the space of renormalized trajectories in theory space. In other words, covariance of the VF demands the introduction of finite counterterms $c_{i}(g)$ which transform as a connection

$$
c_{i}(g) \rightarrow c_{i}^{\prime}(g)=N(g)\left(\partial_{i}+c_{i}(g)\right) N^{-1}(g),
$$

under the change of the basis given by equation (2.4). This is how connection $c_{i}(g)$ arises naturally in the space of renormalized trajectories[19].

Consistency Conditions of VF with RG Equations and Maxwell Relations

It is crucial to notice that the above VF is assumed to be valid for finite values of the parameters $g^{i}$, i.e., to be valid beyond perturbation theory. This is a reflection of the non-perturbative nature of the conjugacy relation even in field theory. Though the definition of the variational formula is inspired by 
its statistical mechanical counterpart, the relation between a lattice model and the corresponding field theory is non-trivial. Consequently, it is difficult to derive the VF from first principles. Instead, if the existence of the conjugate fields $\mathcal{O}_{i}$, satisfying the conjugacy relation (2.5) and VF (3.2) is assumed, then the consistency of VF can be checked. By demanding consistency with the RG equations (2.3), (2.7), and (2.8) we arrive at the following remarkable relation [16]

$$
\operatorname{vol}\left(S^{D-1}\right) H_{i}^{(s)}(g)=\frac{\partial}{\partial g^{i}} \Psi(g)+\left[c_{i}(g), \Psi(g)\right]+\beta^{j}(g) \Omega_{j i}(g)
$$

where

$$
\Psi(g) \equiv \Gamma(g)+\beta^{i}(g) c_{i}(g)
$$

is a covariant tensor field,

$$
H_{i}^{(s)}(g) \equiv \bar{C}_{i}^{(s)}(r=1 ; g)
$$

and the curvature $\Omega_{i j}$ is defined by

$$
\Omega_{i j}(g) \equiv \partial_{i} c_{j}(g)-\partial_{j} c_{i}(g)+\left[c_{i}(g), c_{j}(g)\right]
$$

The curvature could be determined by demanding the consistency of the VF, when used recursively to evaluate higher order derivatives. This means that we want the VF to satisfy the Maxwell relations:

$$
\partial_{i} \partial_{j}=\partial_{j} \partial_{i}
$$

Imposing these conditions on the second order VF for correlation functions gives an expression for the curvature in terms of a double integral over a finite domain [16]:

$$
\Omega_{i j}(g)\langle\Phi\rangle_{g}=\int_{1 \geq r} d^{D} r \text { F.P. } \int_{1 \geq r^{\prime}} d^{D} r^{\prime}\left\langle\mathcal{O}_{j}^{c}(r)\left(\mathcal{O}_{i}^{c}\left(r^{\prime}\right)-C_{i}\left(r^{\prime}\right)\right) \Phi(0)-(i \leftrightarrow j)\right\rangle_{g}
$$

where F.P. denotes taking the integrable part with respect to $r$. The commutativity condition for more than two derivatives reduces to the Maxwell relations (3.10) and does not give any new conditions.

Consistency condition (3.6) gives a geometrical expression for the singular part of the OPE coefficients; the vector field $\beta^{i}(g)$ (beta functions), the rank-two tensor field $\Psi(g)$ (scale and anomalous dimensions), and the connection $c_{i}(g)$ (finite counterterms), determine the unintegrable part of the short-distance singularities of the theory. These are analogues of the well-known relation between the renormalization constants, the beta functions, and the anomalous dimensions in the dimensional regularization with minimal subtraction scheme[22]. 


\subsection{Variational Formula for OPE Coefficients}

Even though the above VF for correlation functions is assumed to be true non-perturbatively (for arbitrary values of $g$ ), it is hard to use in practical calculations. However it is naturally suited for calculating off-critical correlation functions around a non-trivial fixed point by using only the information about the correlation functions at the fixed point $(g=0)$, where they are easier to calculate because of invariance under scale and sometimes larger symmetry group transformations. This is nothing other than perturbation theory around a UV fixed point, and therefore requires the introduction of a realization of derivatives with respect to the parameters of the theory, valid particularly at the point $g=0$ [3].

The non-analytic dependence of the correlation functions on the parameters shows up in the VF as IR divergences at $g=0$. But by virtue of (2.18) all the non-analytic dependence of correlation functions are encoded in one-point functions. Therefore we have to look for a VF for OPE coefficients which, by assumption, are analytic in parameters and perturbatively calculable. This way of doing perturbative calculations is in the spirit of ref. [10] and more general than the standard diagrammatic way.

In ref. [17], using the VF of correlation functions, a VF formula for the OPE coefficients in the product of two operators was given. Along the same line using the VF (3.2) for the derivatives of correlation functions on both side of OPE (2.11), we obtain the following VF for general OPE coefficientst]

$$
\begin{aligned}
& -\partial_{i} C_{a_{1} \cdots a_{n}}{ }^{b}\left(r_{1}, \cdots, r_{n} ; g\right)\left\langle\phi_{b}\right\rangle_{g}= \\
& \lim _{\epsilon \rightarrow 0}\left[\int_{\substack{\left|r^{\prime}-r_{i}\right| \geq \epsilon \\
r^{\prime} \geq \epsilon}} d^{D} r^{\prime}\left\langle\mathcal{O}_{i}^{c}\left(r^{\prime}\right)\left(\phi_{a_{1}}\left(r_{1}\right) \cdots \phi_{a_{n}}\left(r_{n}\right)-C_{a_{1} \cdots a_{n}}{ }^{b}\left(r_{1}, \cdots, r_{n}\right) \phi_{b}(0)\right)\right\rangle_{g}\right. \\
& +\left(\sum_{k=1}^{n} A_{i a_{k}}{ }^{d}(\epsilon) C_{a_{1} \cdots a_{k-1}} d a_{k+1} \cdots a_{n}\right. \\
& b
\end{aligned}
$$

To be consistent with the differentiability of OPE coefficients, the above VF should be free of IR divergences. This consistency is automatic because from OPE (2.11), for any $R^{\prime}>\max \left\{r_{1}, \cdots r_{n}\right\}$, we have

$$
\int_{r^{\prime} \geq R^{\prime}} d^{D} r^{\prime}\left\langle\mathcal{O}_{i}^{c}\left(r^{\prime}\right)\left(\phi_{a_{1}}\left(r_{1}\right) \cdots \phi_{a_{n}}\left(r_{n}\right)-C_{a_{1} \cdots a_{n}}{ }^{b}\left(r_{1}, \cdots, r_{n}\right) \phi_{b}(0)\right)\right\rangle_{g}=0
$$

So if we consider $R^{\prime}$ as the IR cutoff for equation (3.12), the integral is independent of the cutoff. On the other hand, since the integral is performed over a finite domain, it is free of infrared divergences as well. As expected physically, this guarantees that UV physics completely determines the structure of the OPE coefficients; $R^{\prime}$ can be chosen as small as, but not equal to the largest argument of the OPE coefficients.

\footnotetext{
${ }^{13}$ Usually the correlation functions are known not only for the fixed point but also for some special path out of the fixed point. In these cases we can use VF on the trivial path to calculate the correlation functions in its vicinity perturbatively (e.g., In the massive $\left(\phi^{4}\right)_{4}$ theory, since all correlation functions are known for the free massive theory, the VF for $\lambda=0$ could be used to peturbatively calculate the OPE coefficients in the vicinity of the free massive path (17]).

${ }^{14}$ For the rest of this section all formulas are for a finite $g$, but for simplicity we suppress some of the $g$-dependences.
} 
The VF (3.12) easily generalizes to higher-point functions. We have the following first order VF for general OPE coefficients when $\max \left\{r_{1} \cdots r_{n}\right\}<R^{\prime}<\min \left\{s_{1} \cdots s_{l}\right\}$

$$
\begin{aligned}
& -\partial_{i} C_{a_{1} \cdots a_{n}}{ }^{b}\left(r_{1}, \cdots, r_{n} ; g\right)\left\langle\phi_{b}(0) \phi_{d_{1}}\left(s_{1}\right) \cdots \phi_{d_{l}}\left(s_{l}\right)\right\rangle_{g}= \\
& \lim _{\epsilon \rightarrow 0}\left[\int_{\substack{\left|r^{\prime}-r_{i}\right| \geq \epsilon \\
R^{\prime} \geq r^{\prime} \geq \epsilon}} d^{D} r^{\prime}\left\langle\mathcal{O}_{i}^{c}\left(r^{\prime}\right)\left(\phi_{a_{1}}\left(r_{1}\right) \cdots \phi_{a_{n}}\left(r_{n}\right)-C_{a_{1} \cdots a_{n}}{ }^{b}\left(r_{1}, \cdots, r_{n}\right) \phi_{b}(0)\right) \phi_{d_{1}}\left(s_{1}\right) \cdots \phi_{d_{l}}\left(s_{l}\right)\right\rangle_{g}\right. \\
& \left.+\left(\sum_{k=1}^{n} A_{i a_{k}}{ }^{d}(\epsilon) C_{a_{1} \cdots a_{k-1} d a_{k+1} \cdots a_{n}}{ }^{b}\left(r_{1}, \cdots, r_{n}\right)-C_{a_{1} \cdots a_{n}}{ }^{d}\left(r_{1}, \cdots, r_{n}\right) A_{i d}{ }^{b}(\epsilon)\right)\left\langle\phi_{b}(0) \phi_{d_{1}}\left(s_{1}\right) \cdots \phi_{d_{l}}\left(s_{l}\right)\right\rangle_{g}\right],
\end{aligned}
$$

which is manifestly free of any divergences (both UV and IR).

Higher Order VF and Consistency with Analyticity of OPE Coefficients

We can use the VF recursively to evaluate higher order derivatives of OPE coefficients. The consistency of the higher order VF with the commutativity of the partial derivatives (i.e., Maxwell relations) has already been checked and resulted in an expression for the curvature. This type of consistency is related to the UV behavior of the variational formula; the curvature is in terms of double integral over a finite domain. But we have another consistency check: the VF should be consistent with the analyticity assumption of the OPE coefficients. This is related to the IR behavior of VF. In the following we will show that, like the first order VF, this consistency can be made automatic for higher orders recursively.

To avoid too many indices and to see the structure of higher order VF in a more transparent way, let us consider the OPE coefficients for two fields in the matrix form. Later we will only use this higher order VF for the Ising model. Consider the full OPE for two composite fields

$$
\phi_{a}(r) \Phi(0)=C_{a}(r ; g) \Phi(0)
$$

Consider the following two examples of first order, manifestly finite VFs for $C_{a}$ :

$$
\begin{aligned}
&-\partial_{i} C_{a}(r ; g)\langle\Phi\rangle_{g}=\lim _{\epsilon \rightarrow 0}\left[\int_{\substack{\left|r^{\prime}-r\right| \geq \epsilon \\
R^{\prime} \geq r^{\prime} \geq \epsilon}} d^{D} r^{\prime}\left\langle\mathcal{O}_{i}^{c}\left(r^{\prime}\right)\left(\phi_{a}(r)-C_{a}(r)\right) \Phi(0)\right\rangle_{g}\right.\left.+\left(\left[A_{i}(\epsilon), C_{a}(r)\right]+A_{i a}{ }^{d}(\epsilon) C_{d}(r)\right)\langle\Phi\rangle_{g}\right], \\
&-\partial_{i} C_{a}(r ; g)\left\langle\Phi(0) \phi_{d}(s)\right\rangle_{g}=\lim _{\epsilon \rightarrow 0}\left[\int_{\substack{\left|r^{\prime}-r\right| \geq \epsilon \\
R^{\prime} \geq r^{\prime} \geq \epsilon}} d^{D} r^{\prime}\left\langle\mathcal{O}_{i}^{c}\left(r^{\prime}\right)\left(\phi_{a}(r)-C_{a}(r)\right) \Phi(0) \phi_{d}(s)\right\rangle_{g}\right. \\
&\left.+\left(\left[A_{i}(\epsilon), C_{a}(r)\right]+A_{i a}{ }^{d}(\epsilon) C_{d}(r)\right)\left\langle\Phi(0) \phi_{d}(s)\right\rangle_{g}\right],
\end{aligned}
$$

where $r<R^{\prime}<s$. 
The second order VF could be derived by taking the derivative of VF (3.16) and using the VF for correlation functions (3.2). At the end we have

$$
\begin{gathered}
\partial_{j} \partial_{i} C_{a}(r ; g)\langle\Phi\rangle_{g}=\lim _{\epsilon \rightarrow 0}\left[\int _ { \substack { | r ^ { \prime } - r | \geq \epsilon \\
R ^ { \prime } \geq r ^ { \prime } \geq \epsilon } } d ^ { D } r ^ { \prime } \operatorname { l i m } _ { \eta \rightarrow 0 } \left(\int_{\substack{\left|r^{\prime \prime}-r\right| \geq \eta \\
\mid r^{\prime \prime}-r^{\prime} \geq \geq \\
R^{\prime \prime} \geq r^{\prime \prime} \geq \eta}} d^{D} r^{\prime \prime}\left\langle\mathcal{O}_{j}^{c}\left(r^{\prime \prime}\right) \mathcal{O}_{i}^{c}\left(r^{\prime}\right)\left(\phi_{a}(r)-C_{a}(r)\right) \Phi(0)\right\rangle_{g}\right.\right. \\
+\left(\partial_{j} C_{a}(r)+\left[A_{j}(\eta), C_{a}(r)\right]+A_{j a}{ }^{b}(\eta) C_{b}(r)\right)\left\langle\mathcal{O}_{i}^{c}\left(r^{\prime}\right) \Phi(0)\right\rangle_{g}+A_{j i}{ }^{b}(\eta)\left\langle\phi_{b}^{c}\left(r^{\prime}\right)\left(\phi_{a}(r)-C_{a}(r)\right) \Phi(0)\right\rangle_{g} \\
\left.+A_{j a}{ }^{b}(\eta)\left\langle\mathcal{O}_{i}^{c}\left(r^{\prime}\right)\left(\phi_{b}(r)-C_{b}(r)\right) \Phi(0)\right\rangle_{g}+A_{j}(\eta)\left\langle\mathcal{O}_{i}^{c}\left(r^{\prime}\right)\left(\phi_{a}(r)-C_{a}(r)\right) \Phi(0)\right\rangle_{g}\right) \\
-\partial_{j}\left(\left[A_{i}(\epsilon), C_{a}(r)\right]+A_{i a}{ }^{b}(\epsilon) C_{b}(r)\right)\langle\Phi\rangle_{g}+\left(\partial_{i} C_{a}(r)+\left[A_{i}(\epsilon), C_{a}(r)\right]+A_{i a}{ }^{b}(\epsilon) C_{b}(r)\right) \\
\left.\times \lim _{\eta \rightarrow 0}\left(\int_{R^{\prime \prime} \geq r^{\prime \prime} \geq \eta} d^{D} r^{\prime \prime}\left\langle\mathcal{O}_{j}^{c}\left(r^{\prime \prime}\right) \Phi(0)\right\rangle+A_{j}(\eta)\langle\Phi\rangle_{g}\right)\right],(3.18)
\end{gathered}
$$

where $r<R^{\prime}<R^{\prime \prime}$. It is important to note that in the above formula, and all higher order VFs, whenever two or more connected operators are inserted, partial connectedness with respect to all of the inserted operators is implied.

The $R^{\prime}$ independence of VF (3.18) is guaranteed since our starting point, the first order VF (3.16), is already shown to be independent of $R^{\prime}$ as a direct consequence of the OPE itself (i.e., zero-th order $\mathrm{VF}$ ). In the same spirit we can use the first order VF contracted with two point functions (3.17), to find the following identity

$$
\begin{aligned}
\lim _{\epsilon \rightarrow 0}\left[\int_{\substack{\left|r^{\prime}-r\right| \geq \epsilon \\
R^{\prime} \geq r^{\prime} \geq \epsilon}} d^{D} r^{\prime}\right. & \int_{r^{\prime \prime}>R^{\prime \prime}} d^{D} r^{\prime \prime}\left\langle\mathcal{O}_{j}^{c}\left(r^{\prime \prime}\right) \mathcal{O}_{i}^{c}\left(r^{\prime}\right)\left(\phi_{a}(r)-C_{a}(r)\right) \Phi(0)\right\rangle_{g} \\
& \left.+\left(\partial_{i} C_{a}(r)+\left[A_{i}(\epsilon), C_{a}(r)\right]+A_{i a}^{b}(\epsilon) C_{b}(r)\right) \int_{r^{\prime \prime}>R^{\prime \prime}} d^{D} r^{\prime \prime}\left\langle\mathcal{O}_{j}^{c}\left(r^{\prime \prime}\right) \Phi(0)\right\rangle_{g}\right]=0
\end{aligned}
$$

This implies the $R^{\prime \prime}$ independence of the VF (3.18). So we end up with a second order VF which is independent of IR cutoffs and its integrals are over finite ranges, and therefore manifestly finite.

The above arguments for IR finiteness can be generalized, in a straightforward manner, to higher order VFs for general OPE coefficients recursively. Generally for $n \underline{t h}$ order VF of OPE coefficients we end up with $n$ nested volume integrals with increasing cutoffs from the outer integrals to the inner ones. The resulting expression is independent of the cutoffs, the smallest of which may be chosen as small as, but not equal to the largest arguments of the OPE coefficient. This proves the differentiability, to any order, of all OPE coefficients starting from the VF. In another words, this shows the consistency of the VF with the analyticity of OPE coefficients with imposing a mild but calculationally crucial new condition on the nestedness of the IR cutoffs; this variational formula respects the locality of the theory.

\subsection{Systematic Perturbation Theory around a Fixed Point}

Higher order VFs such as (3.18) give us a compact equation for the derivatives of the OPE coeffi- 
cients. But, we need more refinements to develop a systematic method for perturbative calculations First, we must show how to truncate the infinite sums in (3.18). Second, since calculating the integrals with finite IR cutoffs is generally difficult, we will remove the cutoffs carefully. In this section, the above two refinements will be made simultaneously. It then remains to show that the VFs provide us with sufficient conditions for calculating the relevant quantities. We will explicitly show this to be true for the IM in section 5 .

\section{Truncation of VF for OPE coefficients at the Fixed Point}

On physical grounds, we expect that, in the perturbative calculations around the point $g=0$, the contribution of operators with high enough scale dimensions to variational formula for OPE coefficients should die off in the $g \rightarrow 0$ limit. To show that VFs for OPE coefficients, like equation (3.16), have this property we need to go back to their derivations. To keep the presentation simple, let us again consider the case of the second derivative of OPE for two fields. First note that scale covariance (2.2) at the fixed point gives

$$
\left\langle\phi_{a}\right\rangle=0 \quad \text { if } \quad \phi_{a} \neq \mathbf{1}
$$

Here and in what follows we use the convention that fixed point correlation functions are denoted without any subscripts. Taking derivatives of (3.15) we have

$\partial_{j} \partial_{i} C_{a b}{ }^{d}(r ; g)\left\langle\phi_{d}\right\rangle_{g}=\partial_{j} \partial_{i}\left\langle\phi_{a}(r) \phi_{b}(0)\right\rangle_{g}-\partial_{i} C_{a b}{ }^{d}(r ; g) \partial_{j}\left\langle\phi_{d}\right\rangle_{g}-\partial_{j} C_{a b}{ }^{d}(r ; g) \partial_{i}\left\langle\phi_{d}\right\rangle_{g}-C_{a b}{ }^{d}(r ; g) \partial_{j} \partial_{i}\left\langle\phi_{d}\right\rangle_{g}$.

Because of the basic analyticity assumption for the OPE coefficients the left hand side of equation (3.21) is analytic in $g$ except for $\left\langle\phi_{d}\right\rangle_{g}$. So, using (3.20) the right hand side is regular at $g=0$; all divergences of the terms on the right hand side exactly cancel one another. Hence, (3.21) is well defined even at the fixed point. If we use the VF for the derivatives in equations (3.21), we will get the second order VF (3.18) with the infinite sums over operators with arbitrarily high scale dimensions. Instead, let us see what we can say about the derivatives of one point functions before using the VF. Translational invariance and dimensional analysis considerations imply

$$
\begin{aligned}
\partial_{i}\left\langle\phi_{a}\right\rangle=0 & \text { if } \quad x_{a}>y_{i}, \\
\partial_{j} \partial_{i}\left\langle\phi_{a}\right\rangle=0 & \text { if } \quad x_{a}>y_{i}+y_{j} .
\end{aligned}
$$

Using the VF for correlation functions, equations (3.22) and (3.23) give us a hierarchy of identities involving single and double volume integrals over correlation functions. These integrals do not have any IR cutoffs. Each of these integrals could be split into a main part with an IR cutoff and a correction part which is over the region beyond the IR cutoff. We can use these identities to truncate the VF (3.18) such that only a finite number of terms remain. These identities also provide some restrictions on the choice of finite counterterms [28].

If we consider well-behaved field theories in which we only have a finite number of composite operators with scale dimensions less than any given finite number, then we are only left with a finite

\footnotetext{
${ }^{15}$ The notion of conjugacy in theories with conjugate operator(s) which are singular at $g=0$ is much more intricate 16 .
} 
number of terms in the sums and all other terms could be written in terms of these corrections. Because of the dimensional restrictions and the connectedness of the insertions, the corrections vanish at $g=0$ when we take the nested infinite limit of the cutoffs (from the inner to the outer ones). After all these steps we end up with the following VF at the fixed point

$$
\begin{array}{r}
\partial_{j} \partial_{i} C_{a b} \mathbf{1}(r)=\lim _{R^{\prime} \rightarrow \infty} \lim _{R^{\prime \prime} \rightarrow \infty}\left\{r^{\left(y_{i}+y_{j}-x_{a}-x_{b}\right)} \mathcal{M}_{i j, a b}\left(\frac{R^{\prime}}{r}, \frac{R^{\prime \prime}}{r}\right)-r^{\left(y_{i}+y_{j}-x_{d_{i j}}\right)} C_{a b} d_{i j}(r) \mathcal{S}_{i j, d_{i j}}^{I R}\left(\frac{R^{\prime}}{r}, \frac{R^{\prime \prime}}{r}\right)\right. \\
\left.+\left(r^{\left(y_{i}-x_{d_{i}}\right)} \partial_{j} C_{a b} d_{i}(r) \mathcal{S}_{i, d_{i}}^{I R}\left(\frac{R^{\prime}}{r}\right)-\left(\left(i, R^{\prime}\right) \leftrightarrow\left(j, R^{\prime \prime}\right)\right)\right)\right\},(3.24)
\end{array}
$$

where the sums on the repeated indices are restricted by

$$
\begin{gathered}
x_{d_{i}} \leq y_{i}, \\
x_{d_{i j}} \leq y_{i}+y_{j},
\end{gathered}
$$

and the main term and the two types of IR subtraction integrals are defined as follows:

$$
\begin{aligned}
& \mathcal{M}_{i j, a b}\left(U^{\prime}, U^{\prime \prime}\right)= \\
& r^{\left(x_{i}+x_{j}+x_{a}+x_{b}\right)} \lim _{\zeta \rightarrow 0}\left[\int _ { \substack { | u ^ { \prime } - 1 | \geq \zeta \\
U ^ { \prime } \geq u ^ { \prime } \geq \zeta } } d ^ { D } u ^ { \prime } \operatorname { l i m } _ { \xi \rightarrow 0 } \left(\int_{\substack{\left|u^{\prime \prime}-1\right| \geq \xi \\
\mid u^{\prime \prime}-u^{\prime} \geq \geq \\
U^{\prime \prime} \geq U^{\prime \prime} \geq \xi}} d^{D} u^{\prime \prime}\left\langle\mathcal{O}_{j}^{c}\left(r u^{\prime \prime}\right) \mathcal{O}_{i}^{c}\left(r u^{\prime}\right) \phi_{a}(r) \phi_{b}(0)\right\rangle\right.\right. \\
& \left.+A_{j i}{ }^{e}(r \xi)\left\langle\phi_{e}^{c}\left(r u^{\prime}\right) \phi_{a}(r) \phi_{b}(0)\right\rangle+A_{j a}{ }^{e}(r \xi)\left\langle\mathcal{O}_{i}^{c}\left(r u^{\prime}\right) \phi_{e}(r) \phi_{b}(0)\right\rangle+A_{j b}{ }^{e}(r \xi)\left\langle\mathcal{O}_{i}^{c}\left(r u^{\prime}\right) \phi_{a}(r) \phi_{e}(0)\right\rangle\right) \\
& -\partial_{j}\left(A_{i a}{ }^{e}(r \zeta) C_{e b}{ }^{\mathbf{1}}(r)+A_{i b}{ }^{e}(r \zeta) C_{a e}{ }^{\mathbf{1}}(r)\right)+\left(A_{i a}{ }^{e}(r \zeta) C_{e b}{ }^{d_{j}}(r)+A_{i b}{ }^{e}(r \zeta) C_{a e}{ }^{d_{j}}(r)\right) \\
& \left.\left.\times \lim _{\xi \rightarrow 0}\left(\int_{U^{\prime \prime} \geq u^{\prime \prime} \geq \xi} d^{D} u^{\prime \prime}\left\langle\mathcal{O}_{j}^{c}\left(r u^{\prime \prime}\right) \phi_{d_{j}}(0)\right\rangle+A_{j d_{j}} \mathbf{1}(r \xi)\right)\right]\right\}, \\
& \mathcal{S}_{i, d}^{I R}\left(U^{\prime}\right)=r^{\left(x_{i}+x_{d}\right)} \lim _{\zeta \rightarrow 0}\left[\int_{\substack{\left|u^{\prime}-1\right| \geq \zeta \\
U^{\prime} \geq u^{\prime} \geq \zeta}} d^{D} u^{\prime}\left\langle\mathcal{O}_{i}^{c}\left(r u^{\prime}\right) \phi_{d}(0)\right\rangle+A_{i d} \mathbf{1}(r \zeta)\right], \\
& \mathcal{S}_{i j, d}^{I R}\left(U^{\prime}, U^{\prime \prime}\right)=r^{\left(x_{i}+x_{j}+x_{a}+x_{b}\right)} \lim _{\zeta \rightarrow 0}\left[\int _ { \substack { | u ^ { \prime } - 1 | \geq \zeta \\
U ^ { \prime } \geq u ^ { \prime } \geq \zeta } } d ^ { D } u ^ { \prime } \operatorname { l i m } _ { \xi \rightarrow 0 } \left(\int_{\substack{\left|u^{\prime \prime}-1\right| \geq \xi \\
\left|u^{\prime \prime}-u^{\prime}\right| \geq \xi \\
U^{\prime \prime} \geq U^{\prime \prime} \geq \xi}} d^{D} u^{\prime \prime}\left\langle\mathcal{O}_{j}^{c}\left(r u^{\prime \prime}\right) \mathcal{O}_{i}^{c}\left(r u^{\prime}\right) \phi_{a}(r) \phi_{d}(0)\right\rangle\right.\right. \\
& \left.+A_{j i}{ }^{e}(r \zeta) C_{a b}{ }^{d}(r)\left\langle\phi_{e}^{c}\left(r u^{\prime}\right) \phi_{d}(0)\right\rangle+C_{a b}{ }^{d}(r) A_{j d}{ }^{e}(r \xi)\left\langle\mathcal{O}_{i}^{c}\left(r u^{\prime}\right) \phi_{e}(0)\right\rangle\right) \\
& \left.\left.-C_{a b}{ }^{d}(r) \partial_{j} A_{i d}^{\mathbf{1}}(r \zeta)-C_{a b}{ }^{d}(r) A_{i d}^{e}(r \zeta) \lim _{\xi \rightarrow 0}\left(\int_{U^{\prime \prime} \geq u^{\prime \prime} \geq \xi} d^{D} u^{\prime \prime}\left\langle\mathcal{O}_{j}^{c}\left(r u^{\prime \prime}\right) \phi_{e}(0)\right\rangle+A_{j e}{ }^{\mathbf{1}}(\zeta)\right)\right]\right\},
\end{aligned}
$$

where the range for the sums on the upper indices of the subtractions ( $e$ in the above equations) is restricted by the dimensional constraint in the singular OPEs (3.1). Therefore, (3.24) is a finite (both 
UV and IR) and truncated variational formula at the fixed point. After taking the limits as specified above, we do not have any cutoffs left, neither UV nor IR. The generalization of the above construction to higher orders is straightforward. It is, however, important to notice that the order of the limits is crucial; they do not commute. In addition, it is important that we only need to calculate the integrals for infinitely large IR and small UV cutoffs. The case of the Ising model is an instructive example of these points.

\section{$\underline{\text { Calculational Scheme }}$}

The generality of the finiteness proof, presented above, assures the validity of the following shortcut for writing a truncated variational formula at the fixed point. We truncate the formal expression obtained by repeated differentiation of the OPE and its evaluation at the fixed point. Without loss of generality, consider the case of second order perturbation. From equations (3.21), (3.22), and (3.23) we find

$$
\partial_{j} \partial_{i} C_{a b}{ }^{\mathbf{1}}(r)=\partial_{j} \partial_{i}\left\langle\phi_{a}(r) \phi_{b}(0)\right\rangle-\partial_{j} C_{a b}{ }^{d_{i}}(r) \partial_{i}\left\langle\phi_{d_{i}}\right\rangle-\partial_{i} C_{a b} d_{j}(r) \partial_{j}\left\langle\phi_{d_{j}}\right\rangle-C_{a b} d_{i j}(r) \partial_{j} \partial_{i}\left\langle\phi_{d_{i j}}\right\rangle
$$

Now, we replace the derivatives of the correlation functions with their respective variational formulas in the following special way. Every $\partial_{i}$ represents an integration over the position of the inserted operator $\mathcal{O}_{i}$. The range of integration should be bounded by the same IR cutoff, respecting the nestedness of the cutoffs in the main term. So we arrive at the second order finite and truncated VF (3.24), directly.

Then, after having the VFs up to the desired order, we must go through the following steps. First, we should calculate the UV subtractions (3.4) in terms of beta functions, anomalous dimensions, and finite counterterms using the consistency relation (3.6) and the solution of the RG equation (2.16) for the singular OPE coefficients [17]. Second, we must calculate all the necessary critical correlation functions. This can be hard for non-trivial fixed points, and is usually only possible for fixed point theories invariant under a large symmetry group. Third, we can calculate the volume integrals over these correlation functions with infinitely large IR and small UV cutoffs. This is usually the hardest step. We explicitly implement these steps for the spin-spin correlation function in the Ising model to all orders of perturbation theory. Finally, substituting the above relations into the derived VFs, we end up with a set of algebraic equations in terms of the scaling OPE coefficients, their corrections, anomalous dimensions of the composite operators, and the finite counterterms. After choosing a convention for off-critical composite operators by fixing the finite counterterms, up to the restrictions given by the explicit expression for the curvature (3.11), we expect to have sufficient information to solve these equations. A perturbative expansion of the short-distance expansion of correlation functions follows. This step has been carried out up to third order in our previous work 26].

Let us close this section by addressing a subtle point in using the VF at a fixed point. There are cases where one or more derivatives of some one point functions are non-analytic at the fixed point, but the naive $g \rightarrow 0$ limit of the terms corresponding to these derivatives inside the VF give zero due to the critical OPE algebra. The reason for this apparent inconsistency is that, in order to capture the correct non-analyticity structure of the VF for the critical correlation functions, we must be careful about the relation between the two limits, $g \rightarrow 0$ and $R \rightarrow \infty$, where $R$ is a typical IR cutoff. However, because of our proof for the finiteness of the VF for OPE coefficients, we can take the $g \rightarrow 0$ limit of the correlation functions before evaluating the integrals; the nested structure of the IR limits eliminates the kind of divergences which ordinarily appear as singular expressions. In section 5, we will verify this 
point in the Ising model.

\section{UV Structure of VF for the Ising Model}

In the rest of the paper we concentrate on using VF for the calculation of various off-critical quantities for the two dimensional Ising model (IM). Since there are no dimensionless parameters in $\mathrm{IM}$ (i.e., the theory is super-renormalizable) the structure of UV divergences is relatively simple ${ }^{\mathrm{T}}$. On the other hand, for spin fields the critical IM is not trivial and this makes the calculations of the finite parts and the structure of IR divergences of physical quantities very interesting and non-trivial. In this respect IM shares some of the complications of higher order calculations in other off-critical theories in higher dimensions and especially those in two dimensions near unitary minimal models.

\section{Singular OPE Subtractions}

For determining the necessary subtractions in the VF (with respect to $m$ ), we need the following singular OPE coefficients

$$
\mathcal{O}_{m}(r) \phi_{a}(0)=\bar{C}_{m a}^{(s) b}(r ; m) \phi_{b}(0)+O\left(\frac{1}{r^{2}}\right)
$$

From the general solution for OPE coefficients (2.16), the singular part of $C_{m}$ is given by

$$
C_{m}^{(s)}(r ; m)=\frac{1}{r} G(r ; m) H_{m}^{(s)}(m r) G^{-1}(r ; m)
$$

where the analyticity of OPE coefficients implies that $H_{m}^{(s)}(m)$ is a power series in $m$. This together with (2.35) and the fact that the singular part of the OPE coefficients must be at least as singular as $\frac{1}{r^{2}}$ lead to $H_{m}^{(s)}(m)$ [16] as a finite polynomial in $m$ :

$$
H_{m a}^{(s) b}(m)=\sum_{k=0}^{x_{a}-x_{b}-1} \frac{m^{k}}{k !} \partial_{m} H_{m a}^{(s) b}(0) .
$$

On the other hand, from the consistency relation (3.6) we have

$$
2 \pi H_{m}^{(s)}(m)=\partial_{m} \Gamma(m)+c_{m}(m)+m \partial_{m} c_{m}(m)+\left[c_{m}(m), \Gamma(m)\right]
$$

where we have used $\Omega_{m g_{1}}(m)=0$. Inserting (4.4) in (4.2), we can write the singular OPE coefficients in terms of the finite counterterms and anomalous dimensions. To calculate the subtractions we need to calculate the volume integral of the singular OPE coefficients. Note that the matrix $G(r ; m)$ satisfies

\footnotetext{
${ }^{16}$ For a non-trivial example, as far as UV divergences are concerned, consult ref. 17] in which the VF was used to study the perturbatively renormalizable $\left(\phi^{4}\right)_{4}$ theory.
} 


$$
r \frac{\partial}{\partial r} G(r ; m)=-G(r ; m) \Gamma(m r)
$$

We can therefore write the singular OPE coefficients as total derivatives

$$
C_{m}^{(s)}(r ; m)=\frac{1}{2 \pi r} \frac{\partial}{\partial r}\left[G(r ; m)\left(\partial_{m}+r c_{m}(m r)\right) G^{-1}(r ; m)+\pi \beta_{\mathbf{1}} m r^{2}\left(\ln r-\frac{1}{2}\right)\right]
$$

This means that we can calculate the integral in (3.4), and therefore the subtractions can be given in the following closed form

$$
A_{m}(\epsilon ; m)=G(\epsilon ; m)\left(\partial_{m}+\epsilon c_{m}(m \epsilon)\right) G^{-1}(\epsilon ; m) .
$$

Hence, we have an explicit expression for the subtractions in terms of the matirx $G(r ; m)$ and the finite counterterms $c_{m}(m)$ only. Recall that the anomalous dimension matrix $\Gamma(m)$ (2.35) fixes $G(r ; m)$ completely. Therefore, for the case of Ising model, we have completed the first step in the calculational scheme which was outlined at the end of section 3 .

Calculation of the Anomalous Dimension of the Conjugate Operator

Let us consider the VF for the composite operator $\mathcal{O}_{m}$. From (3.2) we have

$$
-\partial_{m}\left\langle\mathcal{O}_{m}\right\rangle_{m}=\lim _{\epsilon \rightarrow 0}\left[\int_{r^{\prime} \geq \epsilon} d^{2} r^{\prime}\left\langle\mathcal{O}_{m}\left(r^{\prime}\right) \mathcal{O}_{m}(0)\right\rangle_{m}^{c}+A_{m m}{ }^{1}(\epsilon ; m)\right],
$$

where from (4.7) and (2.35) we have the following expression for the subtraction

$$
A_{m m}{ }^{1}(\epsilon ; m)=-\beta_{\mathbf{1}} \ln (\epsilon)+c_{m m}{ }^{1}(m) .
$$

Because of the relationship between the scaling limit of the IM and the theory of a free massive Majorana fermion, we have the advantage of having a nonperturbative answer for correlation functions involving only the energy density operator. The off-critical energy density operator is the most general scalar operator of scale dimension one, and in the fermionic representation it is a linear combination of $i \bar{\psi} \psi$ and $m \mathbf{1}$

$$
\mathcal{O}_{m}=\frac{1}{2 \pi}(i \bar{\psi} \psi)+a m \mathbf{1}
$$

This fixes $\mathcal{O}_{m}$ to be $\mathcal{E}=\frac{1}{2 \pi}(i \bar{\psi} \psi)$ at the fixed point. Now from the theory of a free massive Majorana fermion in two dimensions we have 23]

$$
\left\langle\mathcal{O}_{m}(r) \mathcal{O}_{m}(0)\right\rangle_{m}^{c}=\langle i \bar{\psi} \psi(r) \cdot i \bar{\psi} \psi(0)\rangle_{m}^{c}=m^{2}\left(K_{1}^{2}(m r)-K_{0}^{2}(m r)\right)
$$


independent of $a$. The integral in the VF (4.11) can be done analytically and by comparing the result with the derivative of (2.27) with respect to $m$, we find the anomalous dimension of the conjugate operator to be

$$
\beta_{1}=-\frac{1}{2 \pi},
$$

and the integration constant appearing in (2.27), (2.34), and the final expression for the free energy density (2.28) to be related to the finite counterterm as follows

$$
c=\frac{1}{2} \exp \left[\gamma-2 \pi c_{m m}{ }^{1}\right]
$$

where $\gamma$ is the Euler constant. Therefore, upon fixing the finite counterterm $c_{m m}{ }^{\mathbf{1}}$, the integration constant is also fixed.

\section{$\underline{\text { Choice of the Parameters }}$}

The precise definition of the conjugate operator $\mathcal{O}_{m}$ depends on the choice of the parameters $g_{1}$ and $m$ which are by no means unique. We can reparametrize the space of renormalized trajectories by some general mixing of the old parameters up to two general restrictions [15]: The first is that the scale dimensions of the new parameters should be the same as the old ones, and be additively conserved under the RG flow. The second is that, in keeping with the locality of the theory, the reparametrization has to be analytic (i.e., it is given as a power series). So in our case we have only one mixing as follows

$$
\begin{gathered}
\tilde{g}_{\mathbf{1}}=g_{\mathbf{1}}+\frac{\alpha}{2} m^{2}, \\
\tilde{m}=m,
\end{gathered}
$$

where we have kept the normalizations fixed. Under this reparametrization, the conjugate operator and the only relevant finite counterterm change simply as

$$
\begin{gathered}
\tilde{\mathcal{O}}_{m}=\mathcal{O}_{m}-\alpha m \mathbf{1}, \\
\tilde{c}_{m m}{ }^{1}=c_{m m}{ }^{1}+\alpha .
\end{gathered}
$$

Hence, the mixing of $\mathcal{O}_{m}$ with $\mathbf{1}$, or equivalently the change of parameter $g_{1}$, can be controlled by fixing the choice of $c_{m m}{ }^{1}$. Note that, as already discussed in section 2, the correlation functions do not depend on $g_{1}$. This and the fact that any change in $c_{m m}{ }^{1}$ can be absorbed in a change of $g_{1}$, amount to the $c_{m m}{ }^{1}$ independence of the correlation functions.

If we consider off-critical correlation functions which involve only operators corresponding to spin and energy density operators, then for the possible subtractions needed in the VF, $\phi_{a}$ in (4.1) should

\footnotetext{
${ }^{17}$ To find $\beta_{1}$ we do not need to calculate the integral in VF. It is enough to compare (4.9) with the corresponding subtraction coming from the following singular OPE coefficients 26.

$$
\bar{C}_{m m}^{(s) \mathbf{1}}(r ; m)=\frac{1}{4 \pi^{2} r^{2}}
$$
}


be only the spin operator $\sigma$ or the conjugate operator $\mathcal{O}_{m}$ (which respectively correspond to $\sigma$ and $\mathcal{E}$ at the fixed point). In this case, because of the dimensional restriction, the only relevant subtraction is the following which we have already seen in the $\mathrm{VF}$ for $\mathcal{O}_{m}$ :

$$
A_{m m}{ }^{\mathbf{1}}(\epsilon ; m)=\frac{1}{2 \pi} \ln (\epsilon)+c_{m m} \mathbf{1}
$$

This is the main reason that the necessary UV subtractions in VFs for IM are relatively simple, especially in higher orders of perturbation. Generally, this is a feature of super-renormalizable theories. Consider the case of the $n \underline{t h}$ order VF. This means that we have to consider the insertion of volume integral of $\mathcal{O}_{m}^{c} \mathrm{~s}$, and the UV divergent subtractions needed when the inserted operators approach one another or other operators in the correlation function. But, the UV subtractions corresponding to two connected energy density operators are zero. This can be seen in the VF because of the obvious fact that: $\mathbf{1}^{c}=0$. In short; most, but not all, of the UV divergences in higher order perturbative calculations of IM are taken care of by the connectedness of the insertions. The rest of the subtractions require the calculation of the other elements of the anomalous dimension matrix $\Gamma(g)$. $\beta_{\mathbf{1}}$ is the only element needed for the calculation of the short-distance expansion of the spin-spin correlation function to third order 26.

\section{$5 \quad$ Perturbation Theory for the Ising Model}

In this section we implement the calculational scheme, outlined in section 3 , for perturbative analysis of the spin-spin correlation function in the IM in the vicinity of the critical point. After studying the structure of the $n \underline{\underline{t h}} \mathrm{VF}$, we carry out the first two steps of our scheme for the $n^{\underline{t h}}$ order calculation of corrections to scaling. First, we study the structure of the necessary critical correlations. Then, we present a systematic method to calculate the necessary integrals over the critical correlation functions. The final step, which is the fixing of the off-critical operators and solving the resultant algebraic equations, is done up to third order. This section is a systematic extension of the explicit third order calculation done in reference [26], the details of which are presented in appendix B. We leave a systematic study of the final step for general $n \underline{t h}$ order corrections to scaling, for a future work [28].

\section{$5.1 \quad n \underline{t h}$ Order Correction to Scaling}

Consider the short-distance expansion of the spin-spin correlation function. Due to the OPE algebra at the critical point, using the notation of ref.[7] we have

$$
\langle\sigma(r) \sigma(0)\rangle_{m}=C_{\sigma \sigma}^{1 ;\{b\}}(r ; m)\left\langle\mathbf{1}^{\{b\}}\right\rangle_{m}+C_{\sigma \sigma}{ }^{\mathcal{E} ;\{b\}}(r ; m)\left\langle\mathcal{E}^{\{b\}}\right\rangle_{m}
$$

where $\mathbf{1}^{\{b\}}$ and $\mathcal{E}^{\{b\}}$ are the off-critical deformations of the scalar operators in the families $[\mathbf{1}]$ and $[\mathcal{E}]$ (see appendix A). In the above summations, it is enough to consider only those operators which are not total derivatives, since the total derivatives have zero expectation value. 
The OPE algebra of the IM at the fixed point has a $\mathbb{Z}_{2}$ symmetry, known as Kramers-Wannier duality, which implies that the correlation functions involving operators in [1] and an odd number of operators in $[\mathcal{E}]$ are zero. As we have already argued at the end of section 3, this eliminates the contribution of the corresponding derivatives of the one point functions in the VF for the OPE coefficients. Hence, as a natural extension of the results of section 3, using

$$
\partial_{m}^{n}\left\langle\phi_{k}\right\rangle=0 \quad \text { for } n \leq x_{k},
$$

and keeping the subtlety in cancellation of IR divergences in mind, we obtain

$$
\begin{aligned}
& \partial_{m}^{n} C_{\sigma \sigma}{ }^{\mathbf{1}}(r)=\partial_{m}^{n}\langle\sigma(r) \sigma(0)\rangle \\
& -\sum_{\substack{k=1 \\
k \text { even }}}^{n}\left(\begin{array}{l}
n \\
k
\end{array}\right) \sum_{\substack{b \\
x_{b} \leq k}} \partial_{m}^{(n-k)} C_{\sigma \sigma} 1 ;\{b\}(r) \partial_{m}^{k}\left\langle\mathbf{1}^{\{b\}}\right\rangle-\sum_{\substack{k=1 \\
k \text { odd }}}^{n}\left(\begin{array}{l}
n \\
k
\end{array}\right) \sum_{\substack{b \\
x_{b} \leq k}} \partial_{m}^{(n-k)} C_{\sigma \sigma} \mathcal{E} ;\{b\}(r) \partial_{m}^{k}\left\langle\mathcal{E}^{\{b\}}\right\rangle .
\end{aligned}
$$

Note that there are a finite number of operators with $x_{b} \leq n$, and therefore there are a finite number of IR subtractions.

Using the short cut developed in section 3 we can write following finite and truncated $n \underline{t h}$ order VF

$$
\begin{aligned}
& \partial_{m}^{n} C_{\sigma \sigma}{ }^{\mathbf{1}}(r)=\lim _{R_{1} \rightarrow \infty} \cdots \lim _{R_{n} \rightarrow \infty}\left\{(-1)^{n} r^{n-\frac{1}{4}} \mathcal{M}_{n}\left(\frac{R_{1}}{r} \cdots \frac{R_{n}}{r}\right)\right. \\
& -\sum_{\substack{k=1 \\
k \text { even }}}^{n} \sum_{\substack{b \\
x_{b} \leq k}} r^{\left(k-x_{1 ;\{b\}}\right)} \partial_{m}^{(n-k)} C_{\sigma \sigma}{ }^{1 ;\{b\}}(r) \sum_{\substack{\text { perm } \\
\text { from }\left\{p_{1}, \cdots, p_{k}\right\}}} \mathcal{S}_{k, 1 ;\{, \cdots, n\}}^{I R}\left(\frac{R_{p_{1}}}{r} \cdots \frac{R_{p_{k}}}{r}\right) \\
& \left.+\sum_{\substack{k=1 \\
k \text { odd }}}^{n} \sum_{\substack{b \\
x_{b} \leq k}} r^{\left(k-x_{\mathcal{E} ;\{b\}}\right)} \partial_{m}^{(n-k)} C_{\sigma \sigma} \mathcal{E}^{(n ; b\}}(r) \sum_{\substack{\text { perm } \\
\text { from }\left\{p_{1}, \cdots, p_{k}\right\}}} \mathcal{S}_{k, \mathcal{E} ;\{b\}}^{I R}\left(\frac{R_{p_{1}}}{r} \cdots \frac{R_{p_{k}}}{r}\right)\right\},
\end{aligned}
$$

where the main term is

$$
\mathcal{M}_{n}\left(U_{1} \cdots U_{n}\right)=\int_{U_{1} \geq u_{1}} d^{2} u_{1} \cdots \int_{U_{n} \geq u_{n}} d^{2} u_{n} r^{n+\frac{1}{4}}\left\langle\mathcal{E}\left(r u_{n}\right) \cdots \mathcal{E}\left(r u_{1}\right) \sigma(r) \sigma(0)\right\rangle^{c}
$$

and the IR subtraction integrals are

$$
\begin{aligned}
\mathcal{S}_{2 k, 1 ;\{b\}}^{I R}\left(U_{1} \cdots U_{2 k}\right)= & \\
\lim _{\xi_{1} \rightarrow 0} \cdots \lim _{\xi_{k} \rightarrow 0}\left[\int_{U_{1} \geq u_{1} \geq \xi_{1}} d^{2} u_{1} \cdots \int_{U_{2 k} \geq u_{2 k} \geq \xi_{2 k}}\right. & d^{2} u_{2 k} r^{\left(x_{1 ;\{b\}}+2 k\right)}\left\langle\mathcal{E}^{c}\left(r u_{2 k}\right) \cdots \mathcal{E}^{c}\left(r u_{1}\right) \mathbf{1}^{\{b\}}(0)\right\rangle \\
& \left.-\mathcal{S}_{2 k, 1 ;\{b\}}^{U V}\left(\xi_{1}, \cdots \xi_{2 k}, U_{1}, \cdots, U_{2 k}\right)\right],
\end{aligned}
$$

and similar expressions for the IR subtraction integrals $\mathcal{S}_{2 k+1, \mathcal{E} ;\{b\}}^{I R}$. The UV subtractions $\mathcal{S}_{2 k, 1 ;\{b\}}^{U V}$ and $\mathcal{S}_{2 k+1, \mathcal{E} ;\{b\}}^{U V}$, similar to equation (3.29), can be found in terms of $A_{m}(\epsilon)$, its derivatives, and fewer 
volume integrals over correlation functions. The explicit forms of the VFs, up to third order, are given in appendix B.

The VF (5.4) shows the nature of the communication between UV and IR divergences explicitly. The precise statement is that the perturbation for spin-spin correlation function does not have any explicit UV divergences, it involves IR divergences in $\mathcal{M}_{n}$ which show up in the end as non-analytic dependence of the correlation function on the mass. To reveal this structure we need to handle the IR divergences by using the IR subtraction terms, involving $\mathcal{S}_{2 k, 1 ;\{b\}}^{I R}$ and $\mathcal{S}_{2 k+1, \mathcal{E} ;\{b\}}^{I R}$, which are naturally given by OPE. But $\mathcal{S}^{I R}$ S have both IR divergences (which cancel the IR divergences of the main term) and UV divergences (cancelled by the necessary UV subtractions, $\mathcal{S}^{U V} \mathrm{~s}$ ).

\subsection{Calculational Machinery}

\section{$\underline{\text { Critical Correlation Functions }}$}

The free massive Majorana fermion representation for the critical Ising model[ [7] makes the calculation of the energy-density correlation functions trivial. Using the relation (4.10) at $m=0$, calculation of correlation functions involving only the energy-density operators reduces to a product of Pfaffians 23. Then correlation functions of operators in families $[\mathbf{1}]$ and $[\mathcal{E}]$ could be calculated by using the Virasoro algebra. This takes care of all the correlation functions we need for IR subtractions. Note, as an important feature of these correlation functions which makes it possible to calculate their volume integrals, that they do not involve square roots.

The expression of $\sigma(r)$ in terms of the fermion field, on the other hand, is non-local. This makes the calculation of the correlation functions involving the spin operator non-trivial ${ }^{18}$. In two dimensions, due to conformal invariance, all correlation functions are calculable in principle [7]. Moreover, in the case of the Ising model there are additional structures which help in principle to write systematically explicit recursive answers for the correlation functions of the type needed in the main term. Duplicating the IM gives a $c=1$ theory which is amenable to bosonization, and hence the squares of the correlation functions could be calculated. This relationship was recognized much earlier as the equivalence of the Ashkin-Teller model at the end of the self dual line to two non-interacting Ising models 24. Using the above relationships Di Francesco, Saleur, and Zuber found recursive relations for the correlation functions involving energy and spin operators 25]. For correlation functions appearing in the main term (5.5), in terms of a free bosonic field $\phi$ we have

$$
\begin{aligned}
& \left\langle\mathcal{E}\left(r_{n}\right) \cdots \mathcal{E}\left(r_{1}\right) \sigma(r) \sigma(0)\right\rangle\langle\sigma(r) \sigma(0)\rangle=\frac{1}{\pi^{n}}\left\langle\cos 2 \phi\left(r_{n}\right) \cdots \cos 2 \phi\left(r_{1}\right) \cos \phi(r) \cos \phi(0)\right\rangle \\
& -\frac{1}{2} \sum_{k=1}^{n-1} \sum_{\substack{\text { permutation of } \\
\left\{p_{1}, \cdots, p_{n}\right\} \\
\text { from }\{1, \cdots, n\}}}\left\langle\mathcal{E}\left(r_{p_{n}}\right) \cdots \mathcal{E}\left(r_{p_{k+1}}\right) \sigma(r) \sigma(0)\right\rangle\left\langle\mathcal{E}\left(r_{p_{k}}\right) \cdots \mathcal{E}\left(r_{p_{1}}\right) \sigma(r) \sigma(0)\right\rangle .
\end{aligned}
$$

See appendix A for the details and the calculation of correlation functions needed up to third order.

From the above recursive relation and the neutrality condition (A.13) it can be shown inductively that the connected correlation functions have the following structure

\footnotetext{
${ }^{18}$ It is mainly due to this that even though the critical IM has a representation in terms of a free massless Majonara fermion, it is still a non-trivial fixed point.
} 


$$
\left\langle\mathcal{E}\left(r u_{n}\right) \cdots \mathcal{E}\left(r u_{1}\right) \sigma(r) \sigma(0)\right\rangle^{c}=r^{n-\frac{1}{4}} \prod_{k=1}^{n} \frac{1}{2 \pi\left|u_{k}\right|\left|u_{k}-1\right|} \prod_{\substack{p, q=1 \\ p<q}}^{n} \frac{1}{\left|u_{p}-u_{q}\right|^{2}} F_{n}\left(u_{1}, \cdots, u_{n}\right),
$$

where $F_{n}\left(u_{1}, \cdots, u_{n}\right)$ is a real analytic function without any square roots like $\left|u_{p}-u_{q}\right|$. Moreover, $F_{n}$ has the following short-distance structure

$$
F_{n}\left(u_{1}, \cdots, u_{n}\right) \stackrel{u_{p} \rightarrow u_{q}}{\longrightarrow} O\left(u_{p}-u_{q}\right)
$$

Hence, the connected correlation function does not have any unintegrable singularities. Therefore we have the following structure for $\mathcal{M}_{n}$

$$
\mathcal{M}_{n}\left(U_{1}, \cdots, U_{n}\right)=\int_{U_{1} \geq u_{1}} \frac{d^{2} u_{1}}{2 \pi\left|u_{1}\right|\left|u_{1}-1\right|} \cdots \int_{U_{n} \geq u_{n}} \frac{d^{2} u_{n}}{2 \pi\left|u_{n}\right|\left|u_{n}-1\right|} \prod_{\substack{p, q=1 \\ p<q}}^{n} \frac{1}{\left|u_{p}-u_{q}\right|^{2}} F_{n}\left(u_{1}, \cdots, u_{n}\right) .
$$

The need to take the IR limits to infinity in (5.4), gives us a very important calculational advantage: usually it is much easier to calculate integrals for infinitely large cutoffs than for finite ones. This means we are looking for calculation of $\mathcal{M}_{n}$ and $\mathcal{S}^{I R}$, for

$$
1 \ll U_{1} \ll \cdots \ll U_{n} .
$$

Even after the above simplification, the calculation of $\mathcal{M}_{n}$ involves elliptic functions, and beyond first order is extremely hard to do directly. In the following, we develop a systematic way to handle these integrations for any order.

Symmetrization Transformation

For each volume integration in $\mathcal{M}_{n}$ one copy of the integrand of

$$
\mathcal{M}_{1}\left(U_{1}\right)=\int_{U_{1} \geq u_{1}} \frac{d^{2} u_{1}}{4 \pi\left|u_{1}\right|\left|u_{1}-1\right|}
$$

appears. According to the above analysis this is the only source of square roots. Therefore, we can pinpoint the root of the calculational difficulty in the very first order integral $\mathcal{M}_{1}$. The key point in overcoming this difficulty is to use the symmetry properties of the integral. This can be seen by comparing the integral in (5.5) with the ones in the IR subtraction (5.6); in the IR subtraction terms we have both IR and UV divergences. More precisely, the structure of the IR subtractions is invariant under the simultaneous inversion of all variables of the integrals. In the case of the main term only the $\mathcal{M}_{1}$-like factors do not respect this symmetry. The absence of UV divergences in the main term (5.5) is directly related to separation of the singular points in the $\mathcal{M}_{1}$-like part of integrand, one at zero and the other at 1 . Therefore it is enough to look for a transformation which symmetrizes factors of this type. 
This desired transformation changes the complex variable $r_{i}$ with some linear function of $x_{i}+1 / x_{i}$, where $x_{i}$ is the new complex variable. Consider the conformal transformation from $x_{i}$ to $r_{i}$

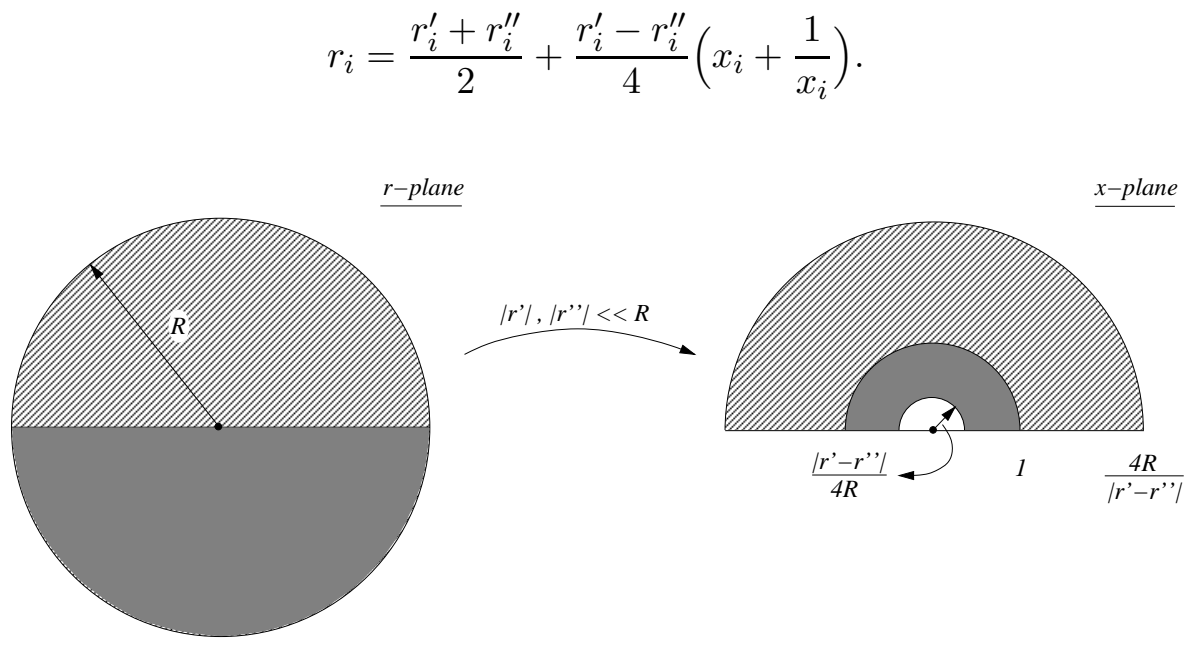

Figure 1: Symmetrization Transformation

which is called the symmetrization transformation [26]. It merges the two integrable singularities in $r_{i}^{\prime}$ and $r_{i}^{\prime \prime}$ to one unintegrable singularity at zero and enforces the symmetry between UV and IR. We only need to calculate the integrals for infinitely large cutoffs as it is given in (5.11). This is crucial in having a simple transformation for the IR cutoffs under the symmetrization (See figure 1). Moreover, using the inversion symmetry which has been produced by symmetrization, we can restrict the domain of integration to the region with radius larger than 1 . So we have the following transformation for a typical integral under symmetrization

$$
\int_{R_{i} \geq r_{i}} \frac{d^{2} r_{i}}{\left|r_{i}-r_{i}^{\prime}\right|\left|r_{i}-r_{i}^{\prime \prime}\right|}=\int_{\frac{4 R_{i}}{\mid r_{i}^{\prime}-r_{i}^{\prime \prime \prime} \geq x_{i} \geq 1}} \frac{d^{2} x_{i}}{\left|x_{i}\right|^{2}}+O\left(\frac{\left|r_{i}^{\prime}\right|}{R_{i}}, \frac{\left|r_{i}^{\prime \prime}\right|}{R_{i}}\right) .
$$

By applying the symmetrization on eq.(5.10) we get the following expression for $\mathcal{M}_{n}$

$$
\begin{aligned}
\mathcal{M}_{n}\left(U_{1}, \cdots, U_{n}\right)= & \int_{4 U_{1} \geq x_{1}} \frac{d^{2} x_{1}}{2 \pi\left|x_{1}\right|^{2}} \cdots \int_{4 U_{n} \geq x_{n}} \frac{d^{2} x_{n}}{2 \pi\left|x_{n}\right|^{2}} \\
& \prod_{\substack{p, q=1 \\
p<q}}^{n} \frac{2\left|x_{p}\right|^{2}\left|x_{q}\right|^{2}}{\left|x_{p}-x_{q}\right|^{2}\left|1-x_{p} x_{q}\right|^{2}} F_{n}\left(\frac{\left(x_{1}+1\right)^{2}}{4 x_{1}}, \cdots, \frac{\left(x_{n}+1\right)^{2}}{4 x_{n}}\right),
\end{aligned}
$$

where we ignore the irrelevant higher order corrections because of the condition (5.11). The symmetrized integrals are much simpler than the integrals we started with. Their integrands are rational functions, and they can be calculated by standard methods. For explicit calculation to the third order see appendix B. 


\section{Spin-Spin-Energy Density Correlation Function Off-criticality}

In this section we demonstrate the applicability of our method for the calculation of more general correlation functions. As a typical example we calculate the short-distance expansion of the spin-spinenergy density correlation function to first order. Our starting point is equation (2.11) for $n=3$

$$
\left\langle\mathcal{E}\left(r^{\prime}\right) \sigma(r) \sigma(0)\right\rangle_{m}=C_{\sigma \sigma m}{ }^{b}\left(r^{\prime}, r ; m\right)\left\langle\phi_{b}\right\rangle_{m},
$$

where in the sum over $b$ it is sufficient to include scalar composite operators, which are not total derivatives. Because of the operator algebra of the critical IM, $\phi_{b} \in\{[\mathbf{1}],[\mathcal{E}]\}$.

Zeroth Order: The Scaling Behavior

At $m=0$, due to equations (3.20) and (A.14), the OPE (6.1) becomes

$$
C_{\sigma \sigma m}{ }^{1}\left(r, r^{\prime}\right)=\frac{r^{\frac{3}{4}}}{4 \pi\left|r^{\prime}-r\right|\left|r^{\prime}\right|} .
$$

$\underline{\text { First Order Corrections to Scaling }}$

The first order variational formula for general OPE coefficients (3.14) can also be truncated. The resulting variational formula can be brought into a more convenient form in which the main term is fully connected. This is motivated by the results of section 4; the necessary UV subtractions are separated out of the main term, and therefore our integration methods are applicable. We can make the main term fully connected by adding and subtracting an appropriate term. Therefore, using the short-hand notation for truncated VFs, we have

$$
\partial_{m} C_{\sigma \sigma m}{ }^{\mathbf{1}}\left(r, r^{\prime}\right)=\partial_{m}\left\langle\mathcal{E}\left(r^{\prime}\right) \sigma(r) \sigma(0)\right\rangle^{c}+\left(\langle\sigma(r) \sigma(0)\rangle-C_{\sigma \sigma m}^{m}\left(r, r^{\prime}\right)\right) \partial_{m}\langle\mathcal{E}\rangle,
$$

which according to the calculational scheme of section 3 is equivalent to

$$
-\partial_{m} C_{\sigma \sigma m}{ }^{\mathbf{1}}\left(r, r^{\prime}\right)=\lim _{R^{\prime \prime} \rightarrow \infty}\left\{r^{-\frac{1}{4}} \mathcal{N}_{1}\left(\frac{r^{\prime}}{r} ; \frac{R^{\prime \prime}}{r}\right)-\left(C_{\sigma \sigma m}^{m}\left(r, r^{\prime}\right)-r^{-\frac{1}{4}}\right)\left(\mathcal{S}_{1, \mathcal{E}}\left(\frac{R^{\prime \prime}}{r}\right)+c_{m m}{ }^{\mathbf{1}}\right)\right\}
$$

where $\mathcal{S}_{1, \mathcal{E}}$ is defined in $(\mathrm{B} .5)$ and

$$
\begin{aligned}
\mathcal{N}_{1}\left(u^{\prime} ; U^{\prime \prime}\right) & \equiv \int_{U^{\prime \prime} \geq u^{\prime \prime}} d^{2} u^{\prime \prime} r^{\frac{9}{4}}\left\langle\mathcal{E}\left(r u^{\prime \prime}\right) \mathcal{E}\left(r u^{\prime}\right) \sigma(r) \sigma(0)\right\rangle^{c} \\
& =\frac{1}{4 \pi\left|u^{\prime}\right|\left|u^{\prime}-1\right|} \int_{4 U^{\prime \prime} \geq u^{\prime \prime}} \frac{d^{2} u^{\prime \prime}}{4 \pi\left|u^{\prime \prime}\right|\left|u^{\prime \prime}-1\right|}\left[\frac{\left|u^{\prime}+u^{\prime \prime}-2 u^{\prime} u^{\prime \prime}\right|^{2}-4\left|u^{\prime}\right|\left|u^{\prime}-1\right|\left|u^{\prime \prime}\right|\left|u^{\prime \prime}-1\right|}{\left|u^{\prime}-u^{\prime \prime}\right|^{2}}\right] .
\end{aligned}
$$

After the symmetrization transformation for infinitely large $U^{\prime \prime}$ we obtain 


$$
\mathcal{N}_{1}\left(\frac{(x+1)^{2}}{4 x} ; U^{\prime \prime}\right)=\frac{4|x|^{2}}{\pi\left|x^{2}-1\right|^{2}} \int_{4 U^{\prime \prime} \geq y \geq 1} \frac{d^{2} y}{4 \pi|y|^{2}} \operatorname{Re}\left[\frac{(x-y)(1-\bar{x} \bar{y})}{(\bar{x}-\bar{y})(1-x y)}\right],
$$

This integral can be calculated with standard techniques. With our previous choice of finite counterterm $c_{m m}{ }^{1}$, the final results are

$$
\begin{gathered}
C_{\sigma \sigma m}^{m}\left(r^{\prime}, r\right)=\frac{\left|2 r^{\prime}-r\right|^{2}}{4\left|r^{\prime}\right|\left|r^{\prime}-r\right| r^{\frac{1}{4}}} \\
\partial_{m} C_{\sigma \sigma m}{ }^{1}\left(r^{\prime}, r\right)=\frac{1}{2 \pi r^{\frac{1}{4}}}\left[\left(\frac{\left|2 r^{\prime}-r\right|^{2}}{4\left|r^{\prime}\right|\left|r^{\prime}-r\right|}+1\right) \ln r-\ln \left(16\left|r^{\prime}\right|\left|r^{\prime}-r\right|\right)+2 \frac{|x|^{4}-1}{\left|x^{2}-1\right|^{2}} \ln |x|\right],
\end{gathered}
$$

where $x=\frac{1}{r}\left(2 r^{\prime}-r \pm 2 \sqrt{r^{\prime}\left(r^{\prime}-r\right)}\right)$. Now inserting back into (6.1) we get

$$
\begin{aligned}
\left\langle\mathcal{E}\left(r^{\prime}\right) \sigma(r) \sigma(0)\right\rangle_{m}= & \frac{r^{\frac{3}{4}}}{4 \pi\left|r^{\prime}-r\right|\left|r^{\prime}\right|}\left[1+\frac{\left|2 r^{\prime}-r\right|^{2} m}{2 r} \ln \left(|m r| e^{\gamma} / 8\right)\right. \\
& \left.\quad-\frac{2\left|r^{\prime}-r \| r^{\prime}\right| m}{r} \ln \left(\frac{16\left|r^{\prime}-r\right|\left|r^{\prime}\right|}{r}\right)+\frac{m r}{4}\left(|x|^{2}-\frac{1}{|x|^{2}}\right) \ln |x|+O\left(m^{2} r^{2}\right)\right] .
\end{aligned}
$$

The two different short-distance limits of this result can be easily checked to be consistent with those obtained from two-point functions already calculated. This is a typical example of the applicability of our calculational scheme for non-trivial higher point functions.

\section{Conclusion and Future Directions}

Three things were done in this paper: First we reviewed the new formalism for the study of the short-distance structure of renormalizable field theories. This formalism provided us with the first systematic scheme for perturbative calculations near a non-trivial fixed point. This review was done both in general and for the special case of the two dimensional massive Ising model at zero external magnetic field. In particular, we presented a complete renormalization group analysis of all the one-point functions of the theory which contain all non-analytic dependence on the mass of any correlation function in the theory. Second, we provided the details of the proof of finiteness of the variational formula for operator product coefficients to all orders. This amounted to the consistency of the variational formula with the analyticity of the OPE coefficients with respect to parameters of the theory. As a by-product, we developed a systematic scheme for perturbative calculations of the OPE coefficients in the vicinity of any fixed point. Our first main result was the truncated and manifestly IR finite $\mathrm{VF}$ (3.24), in which there were only a finite number of terms. This VF can be extended to higher orders readily. Third, we implemented this scheme in the analysis of the short-distance expansion of the Ising model correlation functions which only involve two spin operators. The truncated VF for the case of spin-spin correlation function to $n \frac{t h}{\underline{t h}}$ order was given by equation (5.4). In this case we developed a systematic method for calculating the necessary integrals. Our second main result was the symmetrization transformation (5.13) which is at the heart of our method and transforms these 
extremely hard integrals into a managable form. In particular we calculated the spin-spin and spin-spinenergy density correlation functions to third and first orders, equations (B.16) and (6.9) respectively. Our result for the spin-spin correlation function is in complete agreement with the scaling limit of the well-known exact calculation on the lattice.

This calculational scheme is formulated in coordinate space and its main advantage over conventional schemes is its applicability to perturbative analysis of renormalizable field theories near a non-trivial fixed point. The fundamental strength of this formalism relies on a natural separation of infrared and ultraviolet divergences by incorporating OPE from the outset. An important subtlety to bear in mind is that despite the non-perturbative validity of this formalism, its applicability to perturbative calculations is much more intricate for theories with some ill-behaved conjugate operators at the fixed point [16]. However, our scheme is well-suited for a large class of two dimensional field theories, defined as some perturbation of unitary minimal models [7, 3, 4]. One of the difficult steps in the analysis of these models is to calculate non-trivial finite integrals of the critical correlation functions in each theory. We hope that the possibility of calculating similar integrals in the Ising model sheds some light on the structure of integrals in the more complicated minimal models. Clearly, there is much interesting work to be done.

Among the possible future directions for extending this formalism, three stand out. The first is the renormalization group analysis and perturbative calculation of the correlation functions in the Ising model with non-zero external magnetic field. The second is to understand the structure of the flow between tri-critical and critical Ising models. The third is the completion of the final step of our program for the Ising model, as outlined in section 3. It would be extremely instructive to explicitly see that the validity of our scheme to any order fixes all the anomalous dimensions and the finite counterterms. It should also clarify the role of integrability of the massive Ising model in organizing our field-theoretic calculations effectively[28].

Acknowledgement

We would like to thank H. Sonoda for suggesting the problem, valuable comments, constant encouragement, and a careful reading of the manuscript.

\section{A The Critical Ising Model}

\section{Operator Content}

At the critical point the Ising model acquires conformal symmetry, and we therefore analyze the operator content of the model using the techniques of conformal field theory (Consult [7] for more details.). The local operators of the critical Ising model fall into three classes: class $[\mathbf{1}],[\mathcal{E}]$, and $[\sigma]$. Each class is denoted by their respective primary operators. We call an operator primary if

$$
L_{n} \phi=\bar{L}_{n} \phi=0 \quad \text { for } n>0 \text {, }
$$

where $L_{n}, \bar{L}_{n}(n \in \mathbf{Z})$ are left- and right-moving Virasoro operators. In the class of the primary operator $\phi$ a general operator is given in the following form 


$$
\phi^{\{n\}\{\bar{n}\}} \equiv L_{-n_{1}} \cdots L_{-n_{N}} \bar{L}_{-\bar{n}_{\overline{1}}} \cdots \bar{L}_{-\bar{n}_{\bar{N}}} \phi
$$

To find the linear relation among various operators we need the Virasoro algebra:

$$
\begin{aligned}
& {\left[L_{m}, L_{n}\right]=(m-n) L_{m+n}+\frac{1}{24} m\left(m^{2}-1\right) \delta_{m+n, 0},} \\
& {\left[\bar{L}_{m}, \bar{L}_{n}\right]=(m-n) \bar{L}_{m+n}+\frac{1}{24} m\left(m^{2}-1\right) \delta_{m+n, 0} .}
\end{aligned}
$$

The scale dimension $x$ and the spin $h$ of the local operator $\phi^{\{n\}\{\bar{n}\}}$, are given by:

$$
\begin{aligned}
& x=\left(\Delta+n_{1}+\cdots+n_{N}\right)+\left(\bar{\Delta}+\bar{n}_{\overline{1}}+\cdots+\bar{n}_{\bar{N}}\right), \\
& h=\left(\Delta+n_{1}+\cdots+n_{N}\right)-\left(\bar{\Delta}+\bar{n}_{\overline{1}}+\cdots+\bar{n}_{\bar{N}}\right),
\end{aligned}
$$

where $(\Delta, \bar{\Delta})$ are the eigenvalues of $\left(L_{0}, \bar{L}_{0}\right)$ for $\phi$; for $\mathbf{1}, \mathcal{E}$, and $\sigma$ they are given by $(0,0),\left(\frac{1}{2}, \frac{1}{2}\right)$, and $\left(\frac{1}{16}, \frac{1}{16}\right)$ respectively. Therefore, for scalar operators a lighter notation than the one in (A.2) can be adopted; $\phi^{\{n\}} \equiv \phi^{\{n\}\{n\}}$.

The Ward identity associated with $S L(2, \mathbf{C})$ invariance of conformal field theories implies

$$
L_{-1}=\partial_{z} \equiv\left(\partial_{x}-i \partial_{y}\right) / 2 .
$$

Therefore, using (A.2) and A.3) we find that, up to scale dimension 7, we have the following scalar operators

$$
\mathcal{O}_{0} \equiv \mathbf{1}, \quad \mathcal{O}_{m} \equiv \mathcal{E}, \quad \mathcal{T} \equiv \mathbf{1}^{\{2\}}=L_{-2} \bar{L}_{-2} \mathbf{1}
$$

in the families $[\mathbf{1}]$ and $[\mathcal{E}]$.

The non-trivial operator product expansions between members of these conformal classes are given by:

$$
[\sigma] \cdot[\sigma]=[\mathbf{1}]+[\mathcal{E}], \quad[\sigma] \cdot[\mathcal{E}]=[\sigma], \quad[\mathcal{E}] \cdot[\mathcal{E}]=[\mathbf{1}]
$$

The off-critical Ising model is obtained by adding local scalar operators to the critical hamiltonian density. Therefore the most general hamiltonian for the off-critical Ising model is

$$
\mathcal{H}\left\{\mu_{[\mathbf{1}]}, \mu_{[\mathcal{E}]}, \mu_{[\sigma]}\right\}=\mathcal{H}^{*}+\mu_{[\mathbf{1}]} \int \mathcal{O}_{[\mathbf{1}]}+\mu_{[\mathcal{E}]} \int \mathcal{O}_{[\mathcal{E}]}+\mu_{[\sigma]} \int \mathcal{O}_{[\sigma]}
$$

where the term $\mu_{[i]} \mathcal{O}_{[i]}$ denotes a linear combination of all scalar operators in class $[i]$. By virtue of (A.7) and the fact that RG transformations only depend on local physics we can set $\mu_{[\sigma]}=0$. Note that, since the OPE of two operators in class $[\sigma]$ contains no operators in that class, it make sense to speak of off-critical correlation functions with an even number of spin operators. 


\section{Correlation Functions}

We start by fixing the normalization of our operators at the fixed point

$$
\begin{gathered}
\langle\mathcal{E}(r) \mathcal{E}(0)\rangle=\frac{1}{4 \pi^{2} r^{2 x_{\mathcal{E}}}} \\
\langle\sigma(r) \sigma(0)\rangle=\frac{1}{r^{2 x_{\sigma}}} .
\end{gathered}
$$

The convention that correlation functions have no subscript means that we are calculating fixed point quantities.

We now use the results of [25] to calculate all the necessary correlation functions of the critical Ising model. All the correlation functions are expressed in terms of the correlators of a free bosonic field $\phi$ whose two point function is

$$
\left\langle\phi(r) \phi\left(r^{\prime}\right)\right\rangle=-\frac{1}{2} \ln \left|r-r^{\prime}\right|
$$

which implies

$$
\left\langle e^{i m \phi(r)} e^{i n \phi\left(r^{\prime}\right)}\right\rangle=\left|r-r^{\prime}\right|^{\frac{m n}{2}}
$$

and the neutrality condition

$$
\left\langle\prod_{i=1}^{n} e^{i m_{i} \phi\left(r_{i}\right)}\right\rangle \neq 0 \quad \text { iff } \quad \sum_{i=1}^{n} m_{i}=0 .
$$

The non-trivial three point function is given by (See equation (2.27) of ref. 25])

$$
\left\langle\mathcal{E}\left(r^{\prime}\right) \sigma(r) \sigma(0)\right\rangle_{0}=\frac{1}{\pi}\langle\sigma(r) \sigma(0)\rangle \cdot\left\langle\cos \phi\left(r^{\prime}\right) \cos \phi(r) \cos \phi(0)\right\rangle=\frac{r^{\frac{3}{4}}}{4 \pi\left|r^{\prime}-r \| r^{\prime}\right|} .
$$

By letting $r \rightarrow 0$ and using (A.9) and (A.10) we get:

$$
C_{\sigma \sigma}{ }^{m}(r ; 0)=\pi r^{\frac{3}{4}}
$$

The four point functions of interest are $\langle\mathcal{E} \mathcal{E} \sigma \sigma\rangle$ and $\langle\mathcal{E} \mathcal{E} \mathcal{E} \mathcal{E}\rangle$. Similar to (A.14) we start with (See equation (2.28) of ref. [25])

$$
\begin{array}{r}
\left\langle\mathcal{E}\left(r^{\prime \prime}\right) \mathcal{E}\left(r^{\prime}\right) \sigma(r) \sigma(0)\right\rangle \cdot\langle\sigma(r) \sigma(0)\rangle+\left\langle\mathcal{E}\left(r^{\prime \prime}\right) \sigma(r) \sigma(0)\right\rangle \cdot\left\langle\mathcal{E}\left(r^{\prime}\right) \sigma(r) \sigma(0)\right\rangle \\
=\frac{1}{\pi^{2}}\left\langle\cos 2 \phi\left(r^{\prime \prime}\right) \cos 2 \phi\left(r^{\prime}\right) \cos \phi(r) \cos \phi(0)\right\rangle
\end{array}
$$

\footnotetext{
${ }^{19}$ Note that our particular choice of normalization in $($ A.9 modifies the equations from ref. 25 by factors of $2 \pi$.
} 
and using (A.14), (A.10), and (A.13) we have:

$$
\left\langle\mathcal{E}\left(r^{\prime \prime}\right) \mathcal{E}\left(r^{\prime}\right) \sigma(r) \sigma(0)\right\rangle=\frac{\left|r r^{\prime \prime}+r r^{\prime}-2 r^{\prime \prime} r^{\prime}\right|^{2}}{16 \pi^{2}\left|r^{\prime \prime}\right|\left|r-r^{\prime \prime}\right|\left|r^{\prime}\right|\left|r-r^{\prime}\right|\left|r^{\prime \prime}-r^{\prime}\right|^{2} r^{\frac{1}{4}}} .
$$

from ref. [25] we also have:

$$
\left\langle\mathcal{E}\left(r_{3}\right) \mathcal{E}\left(r_{2}\right) \mathcal{E}\left(r_{1}\right) \mathcal{E}(0)\right\rangle=\sum_{\substack{\text { cyclic permutation } \\ \text { of }\left(r_{3}, r_{2}, r_{1}\right)}} \frac{1}{(2 \pi)^{4}}\left[\frac{\left|r_{1}\right|^{2}\left|r_{2}-r_{3}\right|^{2}}{\left|r_{2}\right|^{2}\left|r_{1}-r_{3}\right|^{2}\left|r_{3}\right|^{2}\left|r_{1}-r_{2}\right|^{2}}-\frac{1}{\left|r_{1}\right|^{2}\left|r_{2}-r_{3}\right|^{2}}\right]
$$

The last correlation function we need is $\langle\mathcal{E} \mathcal{E} \mathcal{E} \sigma \sigma\rangle$. As pointed out in 25], a slight extension of (A.14) and (A.16) allows for calculation of correlation function of any even number of spin operators and an arbitrary number of energy density operators. we therefore have

$$
\begin{array}{r}
\left\langle\mathcal{E}\left(r_{3}\right) \mathcal{E}\left(r_{2}\right) \mathcal{E}\left(r_{1}\right) \sigma(r) \sigma(0)\right\rangle \cdot\langle\sigma(r) \sigma(0)\rangle+\left\langle\mathcal{E}\left(r_{3}\right) \mathcal{E}\left(r_{2}\right) \sigma(r) \sigma(0)\right\rangle \cdot\left\langle\mathcal{E}\left(r_{1}\right) \sigma(r) \sigma(0)\right\rangle \\
+\left\langle\mathcal{E}\left(r_{3}\right) \mathcal{E}\left(r_{1}\right) \sigma(r) \sigma(0)\right\rangle \cdot\left\langle\mathcal{E}\left(r_{2}\right) \sigma(r) \sigma(0)\right\rangle+\left\langle\mathcal{E}\left(r_{2}\right) \mathcal{E}\left(r_{1}\right) \sigma(r) \sigma(0)\right\rangle \cdot\left\langle\mathcal{E}\left(r_{3}\right) \sigma(r) \sigma(0)\right\rangle \\
=8\left\langle\cos 2 \phi\left(r_{3}\right) \cos 2 \phi\left(r_{2}\right) \cos 2 \phi\left(r_{1}\right) \cos \phi(r) \cos \phi(0)\right\rangle
\end{array}
$$

from which, using (A.17), (A.14), (A.10), and (A.13), we get:

$$
\begin{aligned}
\left\langle\mathcal{E}\left(r_{3}\right) \mathcal{E}\left(r_{2}\right) \mathcal{E}\left(r_{1}\right) \sigma(r) \sigma(0)\right\rangle= & \frac{r^{\frac{3}{4}}}{16 \pi^{3}\left|r_{1}\right|\left|r_{1}-r\right|\left|r_{2}\right|\left|r_{2}-r\right|\left|r_{3}\right|\left|r_{3}-r\right|} \\
& \times \sum_{\substack{\text { cyclic permutation } \\
\text { of }\left(r_{3}, r_{2}, r_{1}\right)}}\left[\frac{\left|r_{1}-r_{2}\right|^{2}\left|r_{3}-r\right|^{2}\left|r_{3}\right|^{2}}{\left|r_{3}-r_{1}\right|^{2}\left|r_{3}-r_{2}\right|^{2}}-\frac{\left|r r_{1}+r r_{2}-2 r_{1} r_{2}\right|^{2}}{4\left|r_{1}-r_{2}\right|^{2}}\right] .
\end{aligned}
$$

More general correlation functions can be calculated similarly.

\section{B Calculation of Spin-Spin Correlation Function to Third Order}

In this appendix we present the details of the calculations of ref. [26]. This is an application of the analysis of the $n \underline{t h}$ corrections to scaling for the spin-spin correlation function (section 5 ).

Zero-th Order: The Scaling Behavior

As a consequence of $(3.20)$ and $($ A.10 $)$ we have

$$
C_{\sigma \sigma}{ }^{1}(r)=\frac{1}{r^{1 / 4}}
$$


Corrections to Scaling

For $n=1,2,3$, the equation (5.4) reduces to

$$
\begin{gathered}
-\partial_{m} C_{\sigma \sigma}{ }^{1}(r)=\lim _{R^{\prime} \rightarrow \infty}\left\{r^{\frac{3}{4}} \mathcal{M}_{1}\left(\frac{R^{\prime}}{r}\right)-C_{\sigma \sigma}{ }^{m}(r)\left[\mathcal{S}_{1, \mathcal{E}}\left(\frac{R^{\prime}}{r}\right)+c_{m m}{ }^{1}\right]\right\}, \\
\partial_{m}^{2} C_{\sigma \sigma}{ }^{1}(r)=\lim _{R^{\prime} \rightarrow \infty} \lim _{R^{\prime \prime} \rightarrow \infty}\left\{r^{\frac{7}{4}} \mathcal{M}_{2}\left(\frac{R^{\prime}}{r}, \frac{R^{\prime \prime}}{r}\right)+\partial_{m} C_{\sigma \sigma}{ }^{m}(r)\left[\mathcal{S}_{1, \mathcal{E}}\left(\frac{R^{\prime}}{r}\right)+\mathcal{S}_{1, \mathcal{E}}\left(\frac{R^{\prime \prime}}{r}\right)+2 c_{m m}{ }^{1}\right]\right\},
\end{gathered}
$$

and

$$
\begin{aligned}
-\partial_{m}^{3} C_{\sigma \sigma}{ }^{1}(r)= & \lim _{R_{1} \rightarrow \infty} \lim _{R_{2} \rightarrow \infty} \lim _{R_{3} \rightarrow \infty}\left\{r^{\frac{11}{4}} \mathcal{M}_{3}\left(\frac{R_{1}}{r}, \frac{R_{2}}{r}, \frac{R_{3}}{r}\right)\right. \\
& \left.-r^{2} C_{\sigma \sigma}{ }^{m}(r) \sum_{\text {cyclic perm. }} \mathcal{S}_{3, \mathcal{E}}\left(\frac{R_{1}}{r}, \frac{R_{2}}{r}, \frac{R_{3}}{r}\right)-\partial_{m}^{2} C_{\sigma \sigma}{ }^{m}(r) \sum_{\text {cyclic perm. }} \mathcal{S}_{1, \mathcal{E}}\left(\frac{R_{1}}{r}\right)\right\}
\end{aligned}
$$

where the expression for $\mathcal{M}_{n},(n=1,2,3)$ is given by (5.5) and the subtractions are as follows

$$
\begin{gathered}
\mathcal{S}_{1, \mathcal{E}}\left(U_{1}\right)=\lim _{\epsilon \rightarrow 0}\left(\int_{U_{1} \geq u_{1} \geq \frac{\epsilon}{r}} \frac{d^{2} u_{1}}{4 \pi^{2}\left|u_{1}\right|^{2}}+\frac{1}{2 \pi} \ln (\epsilon)\right), \\
\mathcal{S}_{3, \mathcal{E}}\left(U_{1}, U_{2}, U_{3}\right)=\int_{U_{1} \geq u_{1}} \frac{d^{2} u_{1}}{2 \pi\left|u_{1}\right|^{2}} \int_{U_{2} \geq u_{2}} \frac{d^{2} u_{2}}{2 \pi\left|u_{2}\right|^{2}} \int_{U_{3} \geq u_{3}} \frac{d^{2} u_{3}}{2 \pi\left|u_{3}\right|^{2}}\left[\frac{\left|u_{1}\right|^{4}\left|u_{2}-u_{3}\right|^{2}}{2 \pi\left|u_{1}-u_{3}\right|^{2}\left|u_{1}-u_{2}\right|^{2}}-\frac{\left|u_{2}\right|^{2}\left|u_{3}\right|^{2}}{2 \pi\left|u_{2}-u_{3}\right|^{2}}\right] .
\end{gathered}
$$

The explicit expressions for necessary correlation functions are given in appendix A. For the calculations of the main terms we use the symmetrization transformation (5.13). The resulting integrals are

$$
\begin{gathered}
\mathcal{M}_{1}\left(U_{1}\right)=\int_{4 U_{1} \geq x_{1} \geq 1} \frac{d^{2} x_{1}}{4 \pi\left|x_{1}\right|^{2}}, \\
\mathcal{M}_{2}\left(U_{1}, U_{2}\right)=\int_{4 U_{1} \geq x_{1} \geq 1} \frac{d^{2} x_{1}}{4 \pi\left|x_{1}\right|^{2}} \int_{4 U_{2} \geq x_{2} \geq 1} \frac{d^{2} x_{2}}{4 \pi\left|x_{2}\right|^{2}} R e\left[\frac{\left(x_{1}-x_{2}\right)\left(1-\bar{x}_{1} \bar{x}_{2}\right)}{\left(\bar{x}_{1}-\bar{x}_{2}\right)\left(1-x_{1} x_{2}\right)}\right] \\
\mathcal{M}_{3}\left(U_{1}, U_{2}, U_{3}\right)= \\
\int_{4 U_{1} \geq x_{1}} \frac{d^{2} x_{1}}{2 \pi\left|x_{1}\right|^{2}} \int_{4 U_{2} \geq x_{2}} \frac{d^{2} x_{2}}{2 \pi\left|x_{2}\right|^{2}} \int_{4 U_{3} \geq x_{3}} \frac{d^{2} x_{3}}{2 \pi\left|x_{3}\right|^{2}} \prod_{p, q=1}^{3} \frac{2\left|x_{p}\right|^{2}\left|x_{q}\right|^{2}}{\left|x_{p}-x_{q}\right|^{2}\left|1-x_{p} x_{q}\right|^{2}} \\
\times \sum_{\substack{\text { cyclic } \\
\text { perm. }}}\left\{\left|x_{1}-x_{2}\right|^{4}\left|1-x_{1} x_{2}\right|^{4}\left|x_{3}-1\right|^{4}-\left|x_{1}-x_{3}\right|^{2}\left|x_{2}-x_{3}\right|^{2}\left|1-x_{1} x_{3}\right|^{2}\left|1-x_{2} x_{3}\right|^{2}\right. \\
\left.\times\left(\left|x_{1}^{2}-1\right|^{2}\left|x_{2}^{2}-1\right|^{2}+2 R e\left(\left(x_{1}-x_{2}\right)^{2}\left(1-\bar{x}_{1} \bar{x}_{2}\right)^{2}\right)\right)\right\} .(\mathrm{B} .
\end{gathered}
$$

These integrals do not have any square roots and they are easily calculable. These allow for the calculation of corrections to the scaling behavior of the OPE coefficients. The results are 


$$
\begin{aligned}
C_{\sigma \sigma}{ }^{m}(r) & =\pi r^{\frac{3}{4}}, \\
\partial_{m} C_{\sigma \sigma}{ }^{1}(r) & =\frac{1}{r^{\frac{3}{4}}} \ln r, \\
\partial_{m} C_{\sigma \sigma}{ }^{m}(r) & =0, \\
\partial_{m}^{2} C_{\sigma \sigma}{ }^{1}(r) & =\frac{r^{\frac{7}{4}}}{8}, \\
\partial_{m}^{2} C_{\sigma \sigma}{ }^{m}(r) & =\frac{\pi r^{\frac{11}{4}}}{8}, \\
\partial_{m}^{3} C_{\sigma \sigma}{ }^{1}(r) & =\frac{3 r^{\frac{11}{4}}}{16} \ln r .
\end{aligned}
$$

The results in equations $(\overline{\mathrm{B} .11})$ and $(\overline{\mathrm{B} .15})$ are due to the particular choice of the finite counterterm $c_{m m}{ }^{1}$. Here $c_{m m}{ }^{1}=\frac{1}{\pi} \ln 2$. Consequently from (4.13) we fix the integration constant $c=\frac{e^{\gamma}}{8}$, where $\gamma$ is the Euler's constant. Recall that the answer for the correlation functions is independent of the choice of the finite counterterms (See section 4). With this information at hand, we arrive at the following expression for the short-distance expansion of the spin-spin correlation function

$$
\langle\sigma(r) \sigma(0)\rangle_{m}=\frac{1}{r^{\frac{1}{4}}}\left(1+\frac{1}{2} t \ln \left(|t| e^{\gamma} / 8\right)+\frac{1}{16} t^{2}+\frac{1}{32} t^{3} \ln \left(|t| e^{\gamma} / 8\right)+O\left(t^{4} \ln ^{2}|t|\right)\right),
$$

where $t=m r$. This is in complete agreement with the results of the scaling limit of the lattice calculation[2].

\section{References}

[1] K. Wilson and J. Kogut, Phys. Rep. 12C(1974) 76.

[2] T.T. Wu, B.M. McCoy, C.A. Tracy, and E. Barouch, Phys. Rev. B13(1976) 316.

[3] A.B. Zamolodchikov, JETP Lett. 43(1986) 730; Sov. J. Phys. 46(1988) 1090; Nucl. Phys. B358(1991) 524.

[4] A.B. Zamolodchikov, Adv. Stud. in Pure Math. 19(1989) 641;

Rev. in Math. Phys. Vol 1, No 2(1990) 197.

[5] A.M. Polyakov, JETP Lett. 12(1970) 381.

J. Polchinski, Nucl. Phys. B303(1988) 226.

[6] M. Green, J. Schwartz, and E. Witten, String Theory Vol I (Cambridge Univ. Press, Cambridge, 1985)

A.M. Polyakov, Gauge Fields and Strings (Harwood Academic Publishers 1987)

[7] A.A. Belavin, A.M. Polyakov, and A.B. Zamolodchikov, Nucl. Phys. B241(1984) 333.

[8] Conformal Invariance and Applications to Statistical Mechanics, eds. C. Itzykson, H. Saleur, and J.B. Zuber (World Scientific, Singapore, 1988) 
[9] D.A. Kastor, E.J. Martinec, and S.H. Shenker, Nucl. Phys. B316(1989) 590.

P. Christe and G. Mussardo, Nucl. Phys. B330(1990) 465.

M. Lassig, G. Mussardo, and J.L. Cardy, Nucl. Phys. B348(1991) 591.

[10] K. Wilson, Phys. Rev. 179(1969) 1499.

[11] F. Wegner, Phys. Rev. B5(1972) 4529.

[12] F. Wegner, J. Phys. A8(1975) 710.

[13] H. Sonoda, Nucl. Phys. B352(1991) 585.

[14] H. Sonoda, Nucl. Phys. B352(1991) 601.

[15] H. Sonoda, Nucl. Phys. B366(1991) 629.

[16] H. Sonoda, Nucl. Phys. B383(1992) 173.

[17] H. Sonoda, Nucl. Phys. B394(1993) 302.

[18] K. Ranganathan, H. Sonoda, and B. Zwiebach, Connections on the State-Space over Conformal field Theories (hep-th 9304053).

[19] H. Sonoda, Connections on the Theory Space (hep-th 9306119).

[20] T.D. Schultz, D.C. Mattis, and E.H. Lieb, Rev. Mod. Phys. 36(1964) 856.

[21] G. Parisi, Statistical Field Theory (Addison-Wesley, Menlo Park, 1988).

[22] G. 't Hooft, Nucl. Phys. B62(1973) 444.

[23] See, for example, C. Itzykson and J.M. Drouffe, Statistical Field Theory Vol. I (Cambridge Univ. Press, Cambridge, 1989)

[24] L.P. Kadanoff, A.C. Brown, Ann. Phys. 121(1979) 318.

[25] P. Di Francesco, H. Saleur, and J.B. Zuber Nucl. Phys. B290(1987) 527.

[26] B. Mikhak and A.M. Zarkesh, The Perturbative Calculation of the Spin-Spin Correlation Function in the Two Dimensional Ising Model UCLA Preprint UCLA/93/TEP/48, hep-th/9312202.

[27] V.I. Dotsenko, Nucl. Phys. B314(1989) 687.

[28] B. Mikhak and A.M. Zarkesh, Work in progress. 